\title{
CUT LOCUS AND OPTIMAL SYNTHESIS IN THE SUB-RIEMANNIAN PROBLEM ON THE GROUP OF MOTIONS OF A PLANE*
}

\author{
YURI L. SACHKOV ${ }^{1}$
}

\begin{abstract}
The left-invariant sub-Riemannian problem on the group of motions (rototranslations) of a plane $\mathrm{SE}(2)$ is considered. In the previous works [Moiseev and Sachkov, ESAIM: COCV, DOI: 10.1051/cocv/2009004; Sachkov, ESAIM: COCV, DOI: 10.1051/cocv/2009031], extremal trajectories were defined, their local and global optimality were studied. In this paper the global structure of the exponential mapping is described. On this basis an explicit characterization of the cut locus and Maxwell set is obtained. The optimal synthesis is constructed.
\end{abstract}

Mathematics Subject Classification. 49J15, 93B29, 93C10, 53C17, 22E30.

Received May 28, 2009.

Published online March 24, 2010.

\section{INTRODUCTION}

This work completes the study of the left-invariant sub-Riemannian problem on the group of motions of a plane $\mathrm{SE}(2)=\mathbb{R}^{2} \ltimes \mathrm{SO}(2)$ started in $[7,10]$. In visual geometric terms, this problem can be stated as follows: given two unit vectors $v_{0}=\left(\cos \theta_{0}, \sin \theta_{0}\right), v_{1}=\left(\cos \theta_{1}, \sin \theta_{1}\right)$ attached respectively at two given points $\left(x_{0}, y_{0}\right)$, $\left(x_{1}, y_{1}\right)$ in the plane, one should find an optimal motion in the plane that transfers the vector $v_{0}$ to the vector $v_{1}$, see Figure 1. The vector can move forward or backward and rotate simultaneously. The required motion should be optimal in the sense of minimal length in the space $(x, y, \theta)$, where $\theta$ is the slope of the moving vector.

The corresponding optimal control problem reads as follows:

$$
\begin{aligned}
& \dot{x}=u_{1} \cos \theta, \quad \dot{y}=u_{1} \sin \theta, \quad \dot{\theta}=u_{2}, \\
& q=(x, y, \theta) \in M=\mathbb{R}_{x, y}^{2} \times S_{\theta}^{1}, \quad u=\left(u_{1}, u_{2}\right) \in \mathbb{R}^{2}, \\
& q(0)=q_{0}=(0,0,0), \quad q\left(t_{1}\right)=q_{1}=\left(x_{1}, y_{1}, \theta_{1}\right), \\
& l=\int_{0}^{t_{1}} \sqrt{u_{1}^{2}+u_{2}^{2}} \mathrm{~d} t \rightarrow \min ,
\end{aligned}
$$

\footnotetext{
Keywords and phrases. Optimal control, sub-Riemannian geometry, differential-geometric methods, left-invariant problem, group of motions of a plane, rototranslations, cut locus, optimal synthesis.

* The author is partially supported by Russian Foundation for Basic Research, Project No. 09-01-00246-a, and by the Program of Presidium of Russian Academy of Sciences "Mathematical control theory".

${ }^{1}$ Program Systems Institute, Pereslavl-Zalessky, Russia. sachkov@sys.botik.ru
} 


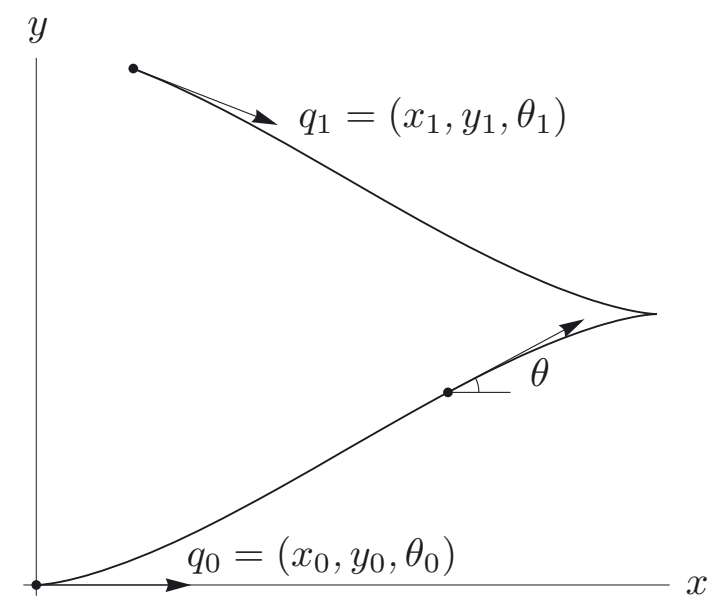

Figure 1. Problem statement.

or, equivalently,

$$
J=\frac{1}{2} \int_{0}^{t_{1}}\left(u_{1}^{2}+u_{2}^{2}\right) \mathrm{d} t \rightarrow \min .
$$

This problem has important relations to vision [4,8,9], robotics [6], and diffusion equation on $\mathrm{SE}(2)$ [2].

Notice that before this work a global description of the cut locus and optimal synthesis was known for left-invariant sub-Riemannian problems on the following Lie groups only: the Heisenberg group (Vershik and Gershkovich [11]), and $\mathrm{SO}(3), \mathrm{SU}(2) \cong S^{3}, \mathrm{SL}(2)$ (Boscain and Rossi [3]).

First we recall the main results of the previous works [7,10]. In paper [7] the normal Hamiltonian system of Pontryagin Maximum Principle was written in a triangular form in appropriate coordinates on cotangent bundle $T^{*} M$, so that its vertical subsystem takes the form of mathematical pendulum:

$$
\begin{aligned}
& \dot{\gamma}=c, \quad \dot{c}=-\sin \gamma, \quad(\gamma, c) \in C \cong\left(2 S_{\gamma}^{1}\right) \times \mathbb{R}_{c} \\
& \dot{x}=\sin \frac{\gamma}{2} \cos \theta, \quad \dot{y}=\sin \frac{\gamma}{2} \sin \theta, \quad \dot{\theta}=-\cos \frac{\gamma}{2}
\end{aligned}
$$

The phase cylinder of pendulum (1.6) decomposes into invariant subsets according to values of the energy $E=c^{2} / 2-\cos \gamma$ :

$$
\begin{aligned}
& C=\bigcup_{i=1}^{5} C_{i}, \\
& C_{1}=\{\lambda \in C \mid E \in(-1,1)\}, \\
& C_{2}=\{\lambda \in C \mid E \in(1,+\infty)\}, \\
& C_{3}=\{\lambda \in C \mid E=1, c \neq 0\}, \\
& C_{4}=\{\lambda \in C \mid E=-1\}=\{(\gamma, c) \in C \mid \gamma=2 \pi n, c=0\}, \\
& C_{5}=\{\lambda \in C \mid E=1, c=0\}=\{(\gamma, c) \in C \mid \gamma=\pi+2 \pi n, c=0\} .
\end{aligned}
$$


In the subsets $C_{1}, C_{2}, C_{3}$ elliptic coordinates $(\varphi, k)$ that rectify the flow of the pendulum were introduced: $\varphi$ is the phase, and $k$ a reparameterized energy of pendulum (1.6):

$$
k=\sqrt{(E+1) / 2} \text { in } C_{1} \cup C_{3}, \quad k=\sqrt{2 /(E+1)} \text { in } C_{2} .
$$

The Hamiltonian system (1.6), (1.7) was integrated in Jacobi's functions [12]. The equation of pendulum (1.6) has a discrete group of symmetries $G=\left\{\operatorname{Id}, \varepsilon^{1}, \ldots, \varepsilon^{7}\right\}=\mathbb{Z}_{2} \times \mathbb{Z}_{2} \times \mathbb{Z}_{2}$ generated by reflections in the axes of coordinates $\gamma, c$, and translations $(\gamma, c) \mapsto(\gamma+2 \pi, c)$. Reflections $\varepsilon^{i}$ are symmetries of the exponential mapping

$$
\operatorname{Exp}: N=C \times \mathbb{R}_{+} \rightarrow M, \quad \operatorname{Exp}(\lambda, t)=q_{t} .
$$

The main result of work [7] is an upper bound on cut time

$$
t_{\text {cut }}=\sup \left\{t_{1}>0 \mid q_{s} \text { is optimal for } s \in\left[0, t_{1}\right]\right\}
$$

along extremal trajectories $q_{s}$. It is based on the fact that a sub-Riemannian geodesic cannot be optimal after a Maxwell point, i.e., a point where two distinct geodesics of equal sub-Riemannian length meet one another. A natural idea is to look for Maxwell points corresponding to discrete symmetries of the exponential mapping. For each extremal trajectory $q_{s}=\operatorname{Exp}(\lambda, s)$, we described Maxwell times $t_{\varepsilon^{i}}^{n}(\lambda), i=1, \ldots, 7, n=1,2, \ldots$, corresponding to discrete symmetries $\varepsilon^{i}$. The following upper bound was proved in work [7]:

$$
t_{\text {cut }}(\lambda) \leq \mathbf{t}(\lambda), \quad \lambda \in C
$$

where $\mathbf{t}(\lambda)=\min \left(t_{\varepsilon^{i}}^{1}(\lambda)\right)$ is the first Maxwell time corresponding to the group of symmetries $G$. We recall the explicit definition of the function $\mathbf{t}(\lambda)$ below in equations (2.1)-(2.5).

In work [10], the local optimality of sub-Riemannian geodesics was completely characterized. Extremal trajectories corresponding to oscillating pendulum (i.e., to $\lambda \in C_{1}$ ) do not have conjugate points, thus they are locally optimal forever. In the case of rotating pendulum $\left(\lambda \in C_{2}\right)$ the first conjugate time is bounded from below and from above by the first Maxwell times $t_{\varepsilon^{2}}^{1}$ and $t_{\varepsilon^{5}}^{1}$ respectively. For critical values of energy of the pendulum, there are no conjugate points. As a consequence, the following bound was proved in Theorem 2.5 [10]:

$$
\mathbf{t}(\lambda) \leq t_{1}^{\mathrm{conj}}(\lambda), \quad \lambda \in C .
$$

Also, in work [10] the global optimality of geodesics was studied. We constructed open dense domains in preimage and image of exponential mapping and proved that the exponential mapping transform these strata diffeomorphically. As a consequence, we showed that inequality (1.13) is in fact an equality.

In this work we obtain our further results for problem (1.1)-(1.5). We consider in detail the action of the exponential mapping at the boundary of the 3-dimensional diffeomorphic domains. This boundary is decomposed into smooth strata of dimension 2, 1, 0 so that restriction of exponential mapping to these strata is a diffeomorphism (Sect. 2). These results provide a detailed description of the global structure of the exponential mapping (Thm. 3.1). The optimal synthesis is constructed in Theorem 3.2.

In Theorems 3.4 and 3.5 we characterize the global structure of the Maxwell set (the set of points $q_{1}$ connected by more than one optimal trajectory with the initial point $q_{0}$ ), and the cut locus (the set of points where extremal trajectories lose optimality). For each point of the Maxwell set there are exactly two optimal trajectories. The cut locus has three connected components $\mathrm{Cut}_{\mathrm{loc}}^{+}$, $\mathrm{Cut}_{\mathrm{loc}}^{-}$, and $\mathrm{Cut}_{\text {glob. }}$. The initial point $q_{0}$ is contained in the closure of the local components $\mathrm{Cut}_{\mathrm{loc}}^{ \pm}$, and is separated from the global component Cutglob. The global component admits a simple description:

$$
\text { Cut }_{\text {glob }}=\{q=(x, y, \theta) \in M \mid \theta=\pi\},
$$


while the local components are subsets of the Moebius strip:

$$
\mathrm{Cut}_{\mathrm{loc}}^{ \pm} \subset\{q=(x, y, \theta) \in M \mid x \cos (\theta / 2)+y \sin (\theta / 2)=0\}
$$

defined by some inequalities, see Theorem 3.5. Embedding of the cut locus in the solid torus is shown at Figure 18.

In Section 4 we present explicit optimal solutions for special boundary conditions.

\section{Structure of exponential mapping at the Boundary of open strata}

We recall some more definitions and notation introduced in the previous works [7,10]. The function $\mathbf{t}$ : $C \rightarrow(0,+\infty]$ on the phase cylinder of pendulum $\left(2 S_{\gamma}^{1}\right) \times \mathbb{R}_{c}=C=\cup_{i=1}^{5} C_{i}$ that evaluates the cut time along sub-Riemannian geodesics $\operatorname{Exp}(\lambda, t), t \in C$, is defined as follows:

$$
\begin{aligned}
& \lambda \in C_{1} \quad \Rightarrow \quad \mathbf{t}(\lambda)=2 K(k), \\
& \lambda \in C_{2} \quad \Rightarrow \quad \mathbf{t}(\lambda)=2 k p_{1}^{1}(k), \\
& \lambda \in C_{3} \quad \Rightarrow \quad \mathbf{t}(\lambda)=+\infty, \\
& \lambda \in C_{4} \quad \Rightarrow \quad \mathbf{t}(\lambda)=\pi, \\
& \lambda \in C_{5} \quad \Rightarrow \quad \mathbf{t}(\lambda)=+\infty,
\end{aligned}
$$

where $p_{1}^{1}(k)$ is the first positive root of the equation $\operatorname{cn} p(\mathrm{E}(p)-p)-\operatorname{dn} p \operatorname{sn} p=0$. Here and below we use Jacobi's functions cn, sn, dn, E, and the complete elliptic integral of the first kind $K$ [12]. Further,

$$
\begin{aligned}
& \widehat{M}=M \backslash\left\{q_{0}\right\}, \\
& \widehat{N}=\{(\lambda, t) \in N \mid t \leq \mathbf{t}(\lambda)\}, \\
& N_{i}=C_{i} \times \mathbb{R}_{+}, \quad i=1, \ldots, 5, \\
& \widetilde{N}=\left\{(\lambda, t) \in \cup_{i=1}^{3} N_{i} \mid t<\mathbf{t}(\lambda), \quad \operatorname{sn} \tau \operatorname{cn} \tau \neq 0\right\}, \\
& N^{\prime}=\left\{(\lambda, t) \in \cup_{i=1}^{3} N_{i} \mid t=\mathbf{t}(\lambda) \text { or } \operatorname{sn} \tau \operatorname{cn} \tau=0\right\} \cup \widehat{N}_{4} \cup N_{5}, \\
& \widehat{N}_{4}=\widehat{N} \cap N_{4}, \\
& \widetilde{M}=\left\{q \in M \mid R_{1}(q) R_{2}(q) \sin \theta \neq 0\right\}, \\
& M^{\prime}=\left\{q \in M \mid R_{1}(q) R_{2}(q) \sin \theta=0\right\},
\end{aligned}
$$

where

$$
R_{1}=y \cos \frac{\theta}{2}-x \sin \frac{\theta}{2}, \quad R_{2}=x \cos \frac{\theta}{2}+y \sin \frac{\theta}{2} .
$$

Along with the coordinates $(k, \varphi, t)$, we use in the domains $N_{1}, N_{2}, N_{3}$ also the coordinates $(k, p, \tau)$ :

$$
\begin{aligned}
& (\lambda, t) \in N_{1} \cup N_{3} \quad \Rightarrow \quad \tau=(2 \varphi+t) / 2, \quad p=t / 2, \\
& (\lambda, t) \in N_{2} \Rightarrow \quad \Rightarrow \quad \tau=(2 \varphi+t) /(2 k), \quad p=t /(2 k) .
\end{aligned}
$$

In work [10] we proved that $\operatorname{Exp}: \widetilde{N} \rightarrow \widetilde{M}$ is a diffeomorphism, and that $\operatorname{Exp}\left(N^{\prime}\right) \subset M^{\prime}$. In this section we describe the action of the exponential mapping

$$
\operatorname{Exp}: N^{\prime}=\widehat{N} \backslash \widetilde{N} \rightarrow M^{\prime}=\widehat{M} \backslash \widetilde{M} .
$$




\subsection{Decomposition of the set $\boldsymbol{N}^{\prime}$}

Consider the following subsets of the set $N^{\prime}$ :

$$
\begin{aligned}
& N_{\text {cut }}=\{(\lambda, t) \in N \mid t=\mathbf{t}(\lambda)\}, \\
& N_{\text {conj }}=\left\{(\lambda, t) \in N_{2} \mid t=\mathbf{t}(\lambda), \quad \text { sn } \tau=0\right\}, \\
& N_{\text {Max }}=N_{\text {cut }} \backslash N_{\text {conj }}, \quad N_{\text {rest }}=N^{\prime} \backslash N_{\text {cut }} .
\end{aligned}
$$

The meaning of the subscripts in $N_{\text {cut }}, N_{\text {conj }}, N_{\text {Max }}$, and $N_{\text {rest }}$ is the following: we will show that $\operatorname{Exp}\left(N_{\text {cut }}\right)$ is the cut locus, $\operatorname{Exp}\left(N_{\text {Max }}\right)$ is the first Maxwell set, $\operatorname{Exp}\left(N_{\text {conj }}\right)$ is the intersection of the cut locus with the conjugate locus (caustic), and $\operatorname{Exp}\left(N_{\text {rest }}\right)$ has no special meaning in this problem (so it contains all the rest strata), see Theorem 3.4. We have the following decompositions:

$$
\widehat{N}=\tilde{N} \sqcup N^{\prime}, \quad N^{\prime}=N_{\text {cut }} \sqcup N_{\text {rest }}, \quad N_{\text {cut }}=N_{\text {Max }} \sqcup N_{\text {conj }},
$$

here and below we denote by $\sqcup$ the union of disjoint sets.

In order to study the structure of the exponential mapping at the set $N^{\prime}$, we need a further decomposition into subsets $N_{i}^{\prime}, i=1, \ldots, 58$, defined by Table 1 .

Images of the projections

$$
\begin{aligned}
& N_{i}^{\prime} \cap\{t<\mathbf{t}(\lambda), \operatorname{sn} \tau \operatorname{cn} \tau=0\} \rightarrow\{p=0\}, \quad(k, \tau, p) \mapsto(k, \tau, 0), \\
& N_{i}^{\prime} \cap\{t=\mathbf{t}(\lambda)\} \rightarrow\{p=0\}, \quad(k, \tau, p) \mapsto(k, \tau, 0),
\end{aligned}
$$

are shown respectively in Figures 2 and 3 .

Table 1 provides a definition of the sets $N_{i}^{\prime}$, e.g., the second column of this table means that

$$
N_{1}^{\prime}=\left\{(\lambda, t) \in N \mid \lambda \in C_{1}^{0}, \tau \in(0, K), p=K, k \in(0,1)\right\} .
$$

Here we use the following decomposition of the sets $C_{i}$ into connected components:

$$
\begin{aligned}
& C_{1}=\cup_{i=0}^{1} C_{1}^{i}, \quad C_{1}^{i}=\left\{(\gamma, c) \in C_{1} \mid \operatorname{sgn}(\cos (\gamma / 2))=(-1)^{i}\right\}, \quad i=0,1, \\
& C_{2}=C_{2}^{+} \cup C_{2}^{-}, \quad C_{2}^{ \pm}=\left\{(\gamma, c) \in C_{2} \mid \operatorname{sgn} c= \pm 1\right\}, \\
& C_{3}=\cup_{i=0}^{1}\left(C_{3}^{i+} \cup C_{3}^{i-}\right), \\
& \quad C_{3}^{i \pm}=\left\{(\gamma, c) \in C_{3} \mid \operatorname{sgn}(\cos (\gamma / 2))=(-1)^{i}, \operatorname{sgn} c= \pm 1\right\}, \quad i=0,1, \\
& C_{4}=\cup_{i=0}^{1} C_{4}^{i}, \quad C_{4}^{i}=\{(\gamma, c) \in C \mid \gamma=2 \pi i, c=0\}, \quad i=0,1, \\
& C_{5}=\cup_{i=0}^{1} C_{5}^{i}, \quad C_{5}^{i}=\{(\gamma, c) \in C \mid \gamma=\pi+2 \pi i, c=0\}, \quad i=0,1 .
\end{aligned}
$$

Introduce the following index sets for numeration of the subsets $N_{i}^{\prime}$ :

$$
\begin{aligned}
& I=\{1, \ldots, 58\}, \quad C=\{1, \ldots, 34\}, \quad J=\{26,28,30,32\} \\
& R=\{35, \ldots, 58\}, \quad X=C \backslash J .
\end{aligned}
$$

Notice that $I=C \sqcup R, J \subset C$.

\section{Lemma 2.1.}

(1) We have $N_{i}^{\prime} \cap N_{j}^{\prime}=\emptyset$ for any distinct $i, j \in I$. 
TABle 1. Definition of sets $N_{i}^{\prime}$.

\begin{tabular}{|c|c|c|c|c|c|c|c|c|}
\hline$N_{i}^{\prime}$ & $N_{1}^{\prime}$ & $N_{2}^{\prime}$ & $N_{3}^{\prime}$ & $N_{4}^{\prime}$ & $N_{5}^{\prime}$ & $N_{6}^{\prime}$ & $N_{7}^{\prime}$ & $N_{8}^{\prime}$ \\
\hline$\lambda$ & $C_{1}^{0}$ & $C_{1}^{0}$ & $C_{1}^{0}$ & $C_{1}^{0}$ & $C_{1}^{1}$ & $C_{1}^{1}$ & $C_{1}^{1}$ & $C_{1}^{1}$ \\
\hline$\tau$ & $(0, K)$ & $(K, 2 K)$ & $(2 K, 3 K)$ & $(3 K, 4 K)$ & $(0, K)$ & $(K, 2 K)$ & $(2 K, 3 K)$ & $(3 K, 4 K)$ \\
\hline$p$ & $K$ & $K$ & $K$ & $K$ & $K$ & $K$ & $K$ & $K$ \\
\hline
\end{tabular}

\begin{tabular}{|c|c|c|c|c|c|c|c|c|}
\hline$N_{i}^{\prime}$ & $N_{9}^{\prime}$ & $N_{10}^{\prime}$ & $N_{11}^{\prime}$ & $N_{12}^{\prime}$ & $N_{13}^{\prime}$ & $N_{14}^{\prime}$ & $N_{15}^{\prime}$ & $N_{16}^{\prime}$ \\
\hline$\lambda$ & $C_{2}^{+}$ & $C_{2}^{+}$ & $C_{2}^{+}$ & $C_{2}^{+}$ & $C_{2}^{-}$ & $C_{2}^{-}$ & $C_{2}^{-}$ & $C_{2}^{-}$ \\
\hline$\tau$ & $(3 K, 4 K$ & $(0, K)$ & $(K, 2 K)$ & $(2 K, 3 K)$ & $(-3 K,-2 K)$ & $(-2 K,-K)$ & $(-K, 0)$ & $(0, K)$ \\
\hline$p$ & $p_{1}^{1}$ & $p_{1}^{1}$ & $p_{1}^{1}$ & $p_{1}^{1}$ & $p_{1}^{1}$ & $p_{1}^{1}$ & $p_{1}^{1}$ & $p_{1}^{1}$ \\
\hline
\end{tabular}

\begin{tabular}{|c|c|c|c|c|c|c|c|c|c|c|c|c|c|c|}
\hline$N_{i}^{\prime}$ & $N_{17}^{\prime}$ & $N_{18}^{\prime}$ & $N_{19}^{\prime}$ & $N_{20}^{\prime}$ & $N_{21}^{\prime}$ & $N_{22}^{\prime}$ & $N_{23}^{\prime}$ & $N_{24}^{\prime}$ & $N_{25}^{\prime}$ & $N_{26}^{\prime}$ & $N_{27}^{\prime}$ & $N_{28}^{\prime}$ & $N_{29}^{\prime}$ & $N_{30}^{\prime}$ \\
\hline$\lambda$ & $C_{1}^{0}$ & $C_{1}^{0}$ & $C_{1}^{0}$ & $C_{1}^{0}$ & $C_{1}^{1}$ & $C_{1}^{1}$ & $C_{1}^{1}$ & $C_{1}^{1}$ & $C_{2}^{+}$ & $C_{2}^{+}$ & $C_{2}^{+}$ & $C_{2}^{+}$ & $C_{2}^{-}$ & $C_{2}^{-}$ \\
\hline$\tau$ & 0 & $K$ & $2 K$ & $3 K$ & 0 & $K$ & $2 K$ & $3 K$ & $3 K$ & 0 & $K$ & $2 K$ & $K$ & $-2 K$ \\
\hline$p$ & $K$ & $K$ & $K$ & $K$ & $K$ & $K$ & $K$ & $K$ & $p_{1}^{1}$ & $p_{1}^{1}$ & $p_{1}^{1}$ & $p_{1}^{1}$ & $p_{1}^{1}$ & $p_{1}^{1}$ \\
\hline
\end{tabular}

\begin{tabular}{|c|c|c|c|c|c|c|c|c|c|c|}
\hline$N_{i}^{\prime}$ & $N_{31}^{\prime}$ & $N_{32}^{\prime}$ & $N_{35}^{\prime}$ & $N_{36}^{\prime}$ & $N_{37}^{\prime}$ & $N_{38}^{\prime}$ & $N_{39}^{\prime}$ & $N_{40}^{\prime}$ & $N_{41}^{\prime}$ & $N_{42}^{\prime}$ \\
\hline$\lambda$ & $C_{2}^{-}$ & $C_{2}^{-}$ & $C_{1}^{0}$ & $C_{1}^{0}$ & $C_{1}^{0}$ & $C_{1}^{0}$ & $C_{1}^{1}$ & $C_{1}^{1}$ & $C_{1}^{1}$ & $C_{1}^{1}$ \\
\hline$\tau$ & $-K$ & 0 & 0 & $K$ & $2 K$ & $3 K$ & 0 & $K$ & $2 K$ & $3 K$ \\
\hline$p$ & $p_{1}^{1}$ & $p_{1}^{1}$ & $(0, K)$ & $(0, K)$ & $(0, K)$ & $(0, K)$ & $(0, K)$ & $(0, K)$ & $(0, K)$ & $(0, K)$ \\
\hline
\end{tabular}

\begin{tabular}{|c|c|c|c|c|c|c|c|c|}
\hline$N_{i}^{\prime}$ & $N_{47}^{\prime}$ & $N_{48}^{\prime}$ & $N_{49}^{\prime}$ & $N_{50}^{\prime}$ & $N_{51}^{\prime}$ & $N_{52}^{\prime}$ & $N_{53}^{\prime}$ & $N_{54}^{\prime}$ \\
\hline$\lambda$ & $C_{3}^{0+}$ & $C_{3}^{0-}$ & $C_{3}^{1+}$ & $C_{3}^{1-}$ & $C_{2}^{+}$ & $C_{2}^{+}$ & $C_{2}^{+}$ & $C_{2}^{+}$ \\
\hline$\tau$ & 0 & 0 & 0 & 0 & $3 K$ & 0 & $K$ & $2 K$ \\
\hline$p$ & $(0,+\infty)$ & $(0,+\infty)$ & $(0,+\infty)$ & $(0,+\infty)$ & $\left(0, p_{1}^{1}\right)$ & $\left(0, p_{1}^{1}\right)$ & $\left(0, p_{1}^{1}\right)$ & $\left(0, p_{1}^{1}\right)$ \\
\hline
\end{tabular}

\begin{tabular}{|c|c|c|c|c|}
\hline$N_{i}^{\prime}$ & $N_{55}^{\prime}$ & $N_{56}^{\prime}$ & $N_{57}^{\prime}$ & $N_{58}^{\prime}$ \\
\hline$\lambda$ & $C_{2}^{-}$ & $C_{2}^{-}$ & $C_{2}^{-}$ & $C_{2}^{-}$ \\
\hline$\tau$ & $K$ & $-2 K$ & $-K$ & 0 \\
\hline$p$ & $\left(0, p_{1}^{1}\right)$ & $\left(0, p_{1}^{1}\right)$ & $\left(0, p_{1}^{1}\right)$ & $\left(0, p_{1}^{1}\right)$ \\
\hline
\end{tabular}

\begin{tabular}{|c|c|c|c|c|c|c|}
\hline$N_{i}^{\prime}$ & $N_{33}^{\prime}$ & $N_{34}^{\prime}$ & $N_{43}^{\prime}$ & $N_{44}^{\prime}$ & $N_{45}^{\prime}$ & $N_{46}^{\prime}$ \\
\hline$\lambda$ & $C_{4}^{0}$ & $C_{4}^{1}$ & $C_{4}^{0}$ & $C_{4}^{1}$ & $C_{5}^{0}$ & $C_{5}^{1}$ \\
\hline$t$ & $\pi$ & $\pi$ & $(0, \pi)$ & $(0, \pi)$ & $(0,+\infty)$ & $(0,+\infty)$ \\
\hline
\end{tabular}

(2) There are the following decompositions of subsets of the set $N^{\prime}$ :

$$
N_{\text {cut }}=\cup_{i \in C} N_{i}^{\prime}, \quad N_{\text {conj }}=\cup_{i \in J} N_{i}^{\prime}, \quad N_{\text {rest }}=\cup_{i \in R} N_{i}^{\prime},
$$

thus

$$
\begin{aligned}
& N_{\text {Max }}=\cup_{i \in X} N_{i}^{\prime}, \\
& N^{\prime}=\sqcup_{i \in I} N_{i}^{\prime} .
\end{aligned}
$$

Proof. Both statements (1) and (2) follow directly from Table 1, definitions of the sets $N^{\prime}, N_{\text {Max }}, N_{\text {cut }}, N_{\text {conj }}$, $N_{\text {rest }}$, and decompositions (2.8). 


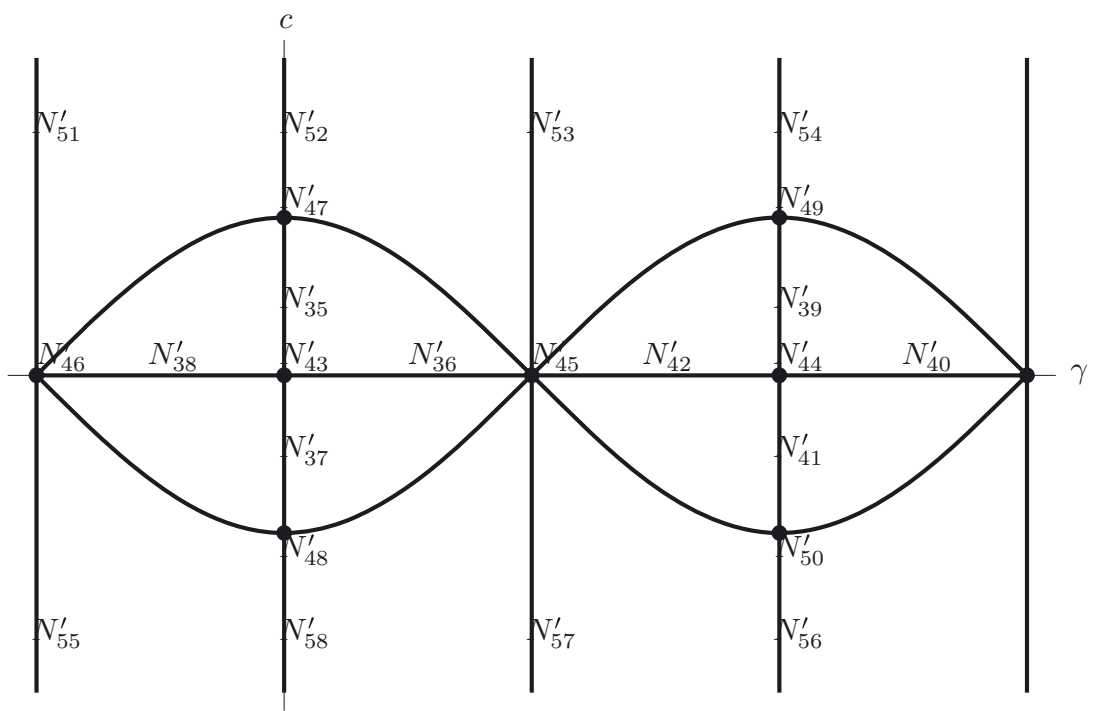

Figure 2. $N_{i}^{\prime} \cap\{t<\mathbf{t}(\lambda), \operatorname{sn} \tau \operatorname{cn} \tau=0\}$.

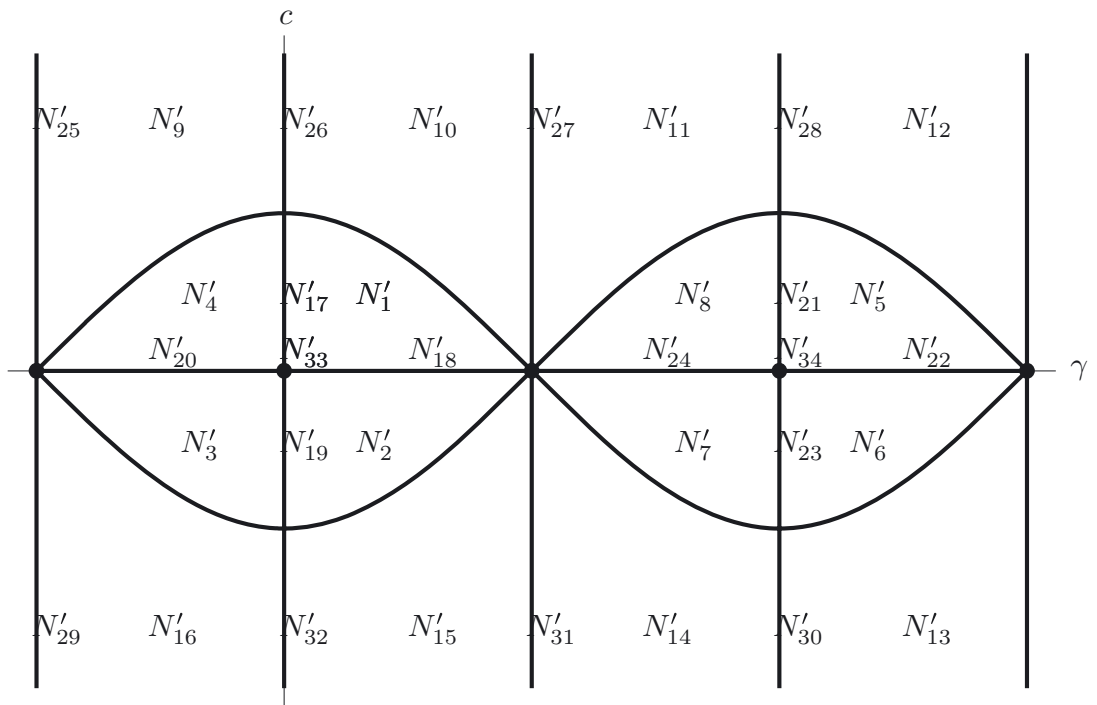

Figure 3. $N_{i}^{\prime} \cap\{t=\mathbf{t}(\lambda)\}$.

\subsection{Exponential mapping of the sets $N_{35}^{\prime}, N_{47}^{\prime}, N_{26}^{\prime}, N_{52}^{\prime}$}

\subsubsection{Exponential mapping of the set $N_{35}^{\prime}$}

In order to describe the image $\operatorname{Exp}\left(N_{35}^{\prime}\right)$, we will need the following function:

$$
R_{1}^{2}(\theta)=2(\operatorname{artanh}(\sin (\theta / 2))-\sin (\theta / 2)), \quad \theta \in[0, \pi) .
$$




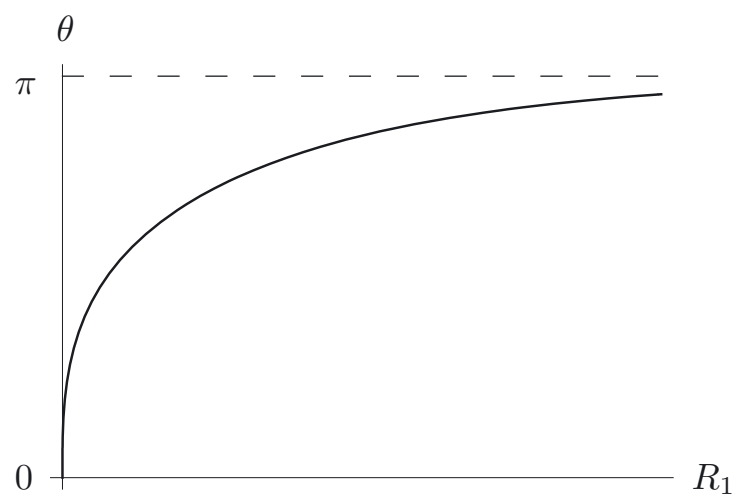

Figure 4. Plot of $R_{1}=R_{1}^{2}(\theta)$.

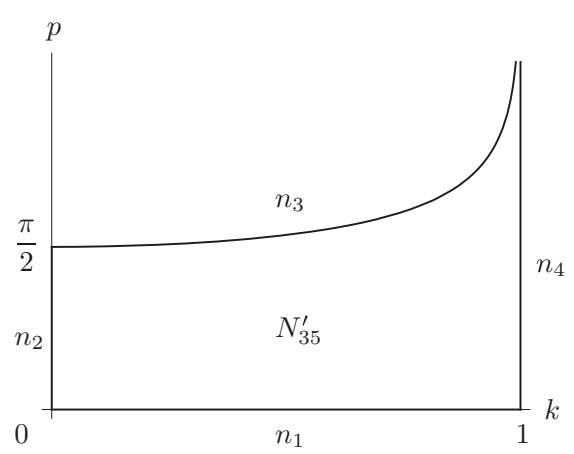

Figure 5. Domain $N_{35}^{\prime}$.

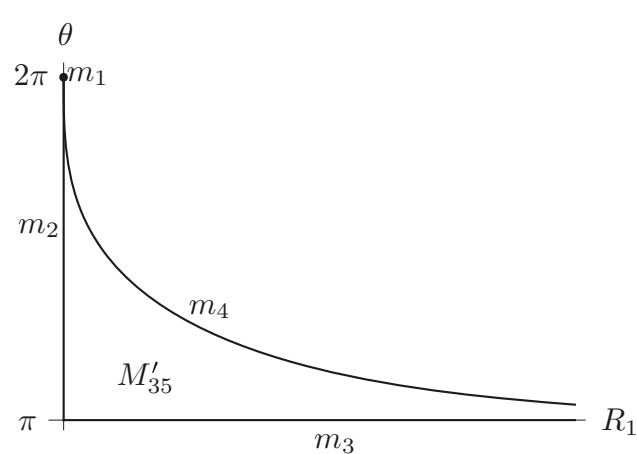

Figure 6. Domain $M_{35}^{\prime}$.

It is obvious that $R_{1}^{2} \in C^{\infty}[0, \pi), R_{1}^{2}(0)=0, R_{1}^{2}(\theta)>0$ for $\theta \in(0, \pi), \lim _{\theta \rightarrow \pi-0} R_{1}^{2}(\theta)=+\infty$, and

$$
\frac{\mathrm{d} R_{1}^{2}}{\mathrm{~d} \theta}(0)=0
$$

A plot of the function $R_{1}^{2}(\theta)$ is given at Figure 4 .

Define the following subset of the set $M^{\prime}$, see Figures 6 and 14:

$$
M_{35}^{\prime}=\left\{q \in M \mid \theta \in(\pi, 2 \pi), R_{1} \in\left(0, R_{1}^{2}(2 \pi-\theta)\right), R_{2}=0\right\} .
$$

Lemma 2.2. The mapping $\operatorname{Exp}: N_{35}^{\prime} \rightarrow M_{35}^{\prime}$ is a diffeomorphism of 2-dimensional manifolds.

To be more precise, we state that $\operatorname{Exp}\left(N_{35}^{\prime}\right)=M_{35}^{\prime}$ and $\left.\operatorname{Exp}\right|_{N_{35}^{\prime}}$ is a diffeomorphism of the manifold $N_{35}^{\prime}$ onto the manifold $M_{35}^{\prime}$. Below we will write such statements briefly as in Lemma 2.2.

Proof. Formulas (5.2)-(5.6) [7] imply that in the domain $N_{35}^{\prime}$ we have the following:

$$
\begin{array}{ll}
\sin (\theta / 2)=\operatorname{sn} p, & \cos (\theta / 2)=-\operatorname{cn} p, \\
R_{1}=2(p-\mathrm{E}(p)) / k, & R_{2}=0 .
\end{array}
$$


By Theorem 2.5 [10] (see (1.14)), the restriction $\left.\operatorname{Exp}\right|_{N_{35}^{\prime}}$ is nondegenerate. Thus the set $\operatorname{Exp}\left(N_{35}^{\prime}\right)$ is an open connected domain in the 2-dimensional manifold

$$
S=\left\{q \in M \mid \theta \in(\pi, 2 \pi), R_{1}>0, R_{2}=0\right\} .
$$

On the other hand, the set $N_{35}^{\prime}$ is an open connected simply connected domain in the 2-dimensional manifold

$$
T=\left\{\nu=(\lambda, t) \in N_{1} \mid \tau=0, p \in(0, K), k \in(0,1)\right\} .
$$

In the topology of $T$, we have

$$
\begin{aligned}
& \partial N_{35}^{\prime}=\cup_{i=1}^{4} n_{i}, \\
& n_{1}=\left\{\nu \in N_{1} \mid \tau=0, p=0, k \in[0,1]\right\}, \\
& n_{2}=\left\{\nu \in N_{1} \mid \tau=0, p \in[0, \pi / 2], k=0\right\}, \\
& n_{3}=\left\{\nu \in N_{1} \mid \tau=0, p=K(k), k \in[0,1)\right\}, \\
& \left.n_{4}=\left\{\nu \in N_{1} \mid \tau=0, p \in[0,+\infty), k=1\right]\right\},
\end{aligned}
$$

see Figure 5.

It follows from formulas (2.15) and (2.16) that

$$
\begin{aligned}
& \operatorname{Exp}\left(n_{1}\right)=m_{1}=\left\{q \in M \mid \theta=2 \pi, R_{1}=0, R_{2}=0\right\}, \\
& \operatorname{Exp}\left(n_{2}\right)=m_{2}=\left\{q \in M \mid \theta \in[\pi, 2 \pi], R_{1}=0, R_{2}=0\right\}, \\
& \operatorname{Exp}\left(n_{3}\right)=m_{3}=\left\{q \in M \mid \theta=\pi, R_{1}>0, R_{2}=0\right\}, \\
& \operatorname{Exp}\left(n_{4}\right)=m_{4}=\left\{q \in M \mid \theta \in[\pi, 2 \pi], R_{1}=R_{1}^{2}(2 \pi-\theta), R_{2}=0\right\},
\end{aligned}
$$

moreover, $\partial M_{35}^{\prime}=\cup_{i=1}^{4} m_{i}$, see Figure 6 .

Now we show that $\operatorname{Exp}\left(N_{35}^{\prime}\right) \subset M_{35}^{\prime}$ and $\operatorname{Exp}: N_{35}^{\prime} \rightarrow M_{35}^{\prime}$ is a diffeomorphism.

(a) We show that $\operatorname{Exp}\left(N_{35}^{\prime}\right) \cap M_{35}^{\prime} \neq \emptyset$. Formulas (2.15) and (2.16) give the following asymptotics as $k \rightarrow 0$ :

$$
\theta=2 \pi-2 p+o(1), \quad R_{1}=k(p / 2-(\sin 2 p) / 4)+o(k) .
$$

There exists $(p, k)$ close to $(\pi / 2,0)$ such that the corresponding point $\left(\theta, R_{1}\right)$ is arbitrarily close to $(0,0)$, with $\theta>0, R_{1}>0$. Thus there exists $\nu \in N_{35}^{\prime}$ such that $\operatorname{Exp}(\nu) \in M_{35}^{\prime}$.

(b) We show that $\operatorname{Exp}\left(N_{35}^{\prime}\right) \neq S$. Formulas (2.15) and (2.16) yield the following chain:

$$
\theta \rightarrow 2 \pi-0 \quad \Rightarrow \quad \sin \rightarrow 0 \quad \Rightarrow \quad p \rightarrow 0 \quad \Rightarrow \quad R_{1} \rightarrow 0 .
$$

Thus there exists $q \in S \backslash \operatorname{Exp}\left(N_{35}^{\prime}\right)$.

(c) We prove that $\operatorname{Exp}\left(N_{35}^{\prime}\right) \subset M_{35}^{\prime}$. By contradiction, suppose that there exists a point $q_{1} \in \operatorname{Exp}\left(N_{35}^{\prime}\right) \backslash M_{35}^{\prime}$. Since the mapping $\left.\operatorname{Exp}\right|_{N_{35}^{\prime}}$ is nondegenerate, we can choose this point such that $q_{1} \in \operatorname{Exp}\left(N_{35}^{\prime}\right) \backslash \operatorname{cl}\left(M_{35}^{\prime}\right)$.

Choose any point $q_{2} \in S \backslash \operatorname{cl}\left(M_{35}^{\prime}\right)$. Connect the points $q_{1}, q_{2}$ by a continuous curve in $S$, and find at this curve a point $q_{3} \in S \backslash \operatorname{Exp}\left(N_{35}^{\prime}\right), q_{3} \notin \operatorname{cl}\left(M_{35}^{\prime}\right)$ such that there exists a converging sequence $q^{n} \rightarrow q_{3}$, $q^{n}=\operatorname{Exp}\left(\nu^{n}\right) \in \operatorname{Exp}\left(N_{35}^{\prime}\right)$. Further, there exist a subsequence $\nu^{n_{i}} \in N_{35}^{\prime}$ converging to a finite or infinite limit. If $\nu^{n_{i}} \rightarrow \bar{\nu} \in N_{35}^{\prime}$, then $q_{3}=\operatorname{Exp}(\bar{\nu}) \in \operatorname{int} \operatorname{Exp}\left(N_{35}^{\prime}\right)$ by nondegeneracy of $\left.\operatorname{Exp}\right|_{N_{35}^{\prime}}$, a contradiction. If $\nu^{n_{i}} \rightarrow \bar{\nu} \in \partial N_{35}^{\prime}$, then

$$
q_{3}=\operatorname{Exp}(\bar{\nu}) \in \operatorname{Exp}\left(\partial N_{35}^{\prime}\right)=\partial M_{35}^{\prime} \subset \operatorname{cl}\left(M_{35}^{\prime}\right),
$$


a contradiction. Finally, if $\nu^{n_{i}} \rightarrow \infty$, then at this sequence $k^{n_{i}} \rightarrow 1-0, p^{n_{i}} \rightarrow \infty$, thus $R_{1}\left(q^{n_{i}}\right) \rightarrow \infty$, a contradiction.

Consequently, $\operatorname{Exp}\left(N_{35}^{\prime}\right) \subset M_{35}^{\prime}$.

(d) The mapping Exp : $N_{35}^{\prime} \rightarrow M_{35}^{\prime}$ is a diffeomorphism since $\left.\operatorname{Exp}\right|_{N_{35}^{\prime}}$ is nondegenerate and proper, and $N_{35}^{\prime}, M_{35}^{\prime}$ are connected and simply connected.

\subsubsection{Exponential mapping of the set $N_{47}^{\prime}$}

Define the following subset of $M^{\prime}$, see Figure 14:

$$
M_{47}^{\prime}=\left\{q \in M \mid \theta \in(\pi, 2 \pi), R_{1}=R_{1}^{2}(2 \pi-\theta), R_{2}=0\right\} .
$$

Lemma 2.3. The mapping Exp : $N_{47}^{\prime} \rightarrow M_{47}^{\prime}$ is a diffeomorphism of 1-dimensional manifolds.

Proof. We pass to the limit $k \rightarrow 1-0$ in formulas (2.15) and (2.16) and obtain for $\nu \in N_{47}^{\prime}$ :

$$
\sin (\theta / 2)=\tanh p, \quad \cos (\theta / 2)=-1 / \cosh p, \quad R_{1}=2(p-\tanh p), \quad R_{2}=0 .
$$

This coordinate representation shows that Exp : $N_{47}^{\prime} \rightarrow M_{47}^{\prime}$ is a diffeomorphism.

\subsubsection{Exponential mapping of the set $N_{26}^{\prime}$}

Before the study of $\left.\operatorname{Exp}\right|_{N_{52}^{\prime}}$, postponed till the next subsection, we need to consider the set $N_{26}^{\prime}$ contained in the boundary of $N_{52}^{\prime}$. In order to parameterize regularly the image $\operatorname{Exp}\left(N_{26}^{\prime}\right)$, we introduce the necessary functions.

Recall that the function $p=p_{1}^{1}(k), k \in[0,1)$, is the first positive root of the function $f_{1}(p)=\operatorname{cn} p(\mathrm{E}(p)-p)-$ $\operatorname{dn} p \operatorname{sn} p$, see equation (5.12) and Corollary 5.1 [7]. Define the function

$$
v_{1}^{1}(k)=\operatorname{am}\left(p_{1}^{1}(k), k\right), \quad k \in[0,1) .
$$

\section{Lemma 2.4.}

(1) The number $v=v_{1}^{1}(k)$ is the first positive root of the function

$$
h_{1}(v, k)=E(v, k)-F(v, k)-\sqrt{1-k^{2} \sin ^{2} v} \tan v, \quad k \in[0,1) .
$$

(2) $v_{1}^{1} \in C^{\infty}[0,1)$.

(3) $v_{1}^{1}(k) \in(\pi / 2, \pi)$ for $k \in(0,1)$; moreover, $v_{1}^{1}(0)=\pi$.

(4) The function $v_{1}^{1}(k)$ is strictly decreasing at the segment $k \in[0,1)$.

(5) $\lim _{v \rightarrow 1-0} v_{1}^{1}(k)=\pi / 2$, thus setting $v_{1}^{1}(1)=\pi / 2$, we obtain $v_{1}^{1} \in C[0,1]$.

(6) $v_{1}^{1}(k)=\pi-(\pi / 2) k^{2}+o\left(k^{2}\right), k \rightarrow+0$.

Proof. (1) follows from (2.17) since $p=p_{1}^{1}$ is the first positive root of the function $f_{1}(p)$.

(2) follows since $p_{1}^{1} \in C^{\infty}[0,1)$ by Lemma $5.3[7]$.

(3) follows since $p_{1}^{1} \in(K, 2 K)$ and $p_{1}^{1}(0)=\pi$, see Corollary 5.1 [7].

(4) We have for $v \in(\pi / 2, \pi]$ :

$$
\begin{aligned}
& \frac{\partial h_{1}}{\partial v}=-\sqrt{1-k^{2} \sin ^{2} v} / \cos ^{2} v<0, \\
& \frac{\partial h_{1}}{\partial k}=-\frac{k}{1-k^{2}}\left(E(v, k)-\sqrt{1-k^{2} \sin ^{2} v} \tan v\right)<0 .
\end{aligned}
$$

Thus $\frac{\mathrm{d} v_{1}^{1}}{\mathrm{~d} k}=-\frac{\partial h_{1} / \partial k}{\partial h_{1} / \partial v}<0$ for $k \in[0,1)$. 


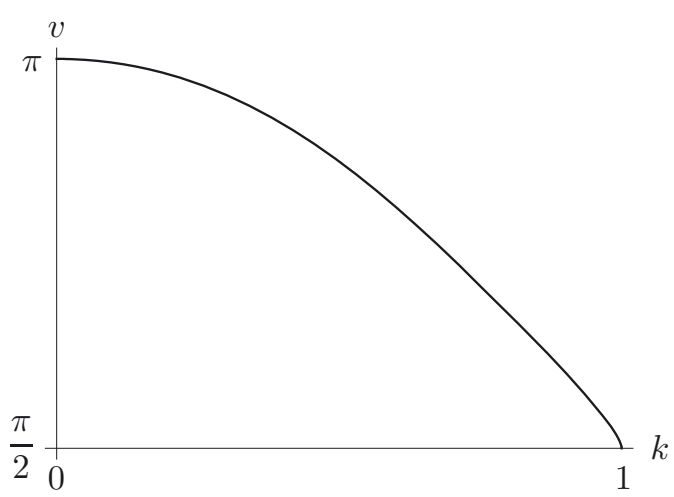

Figure 7. Plot of $v=v_{1}^{1}(k)$.

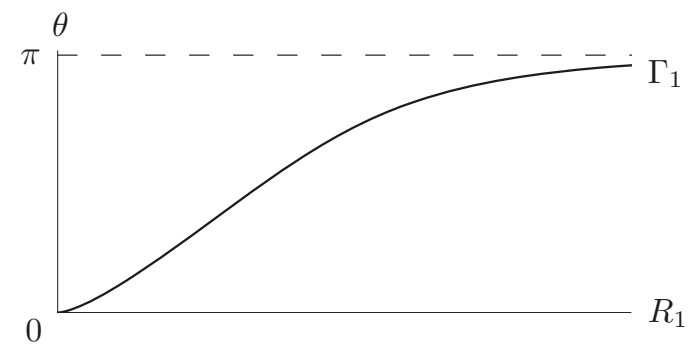

Figure 8. The curve $\Gamma_{1}$.

(5) Monotonicity and boundedness of $v_{1}^{1}(k)$ imply that there exists a limit $\lim _{k \rightarrow 1-0} v_{1}^{1}(k)=\bar{v} \in[\pi / 2, \pi)$. If $\bar{v} \in(\pi / 2, \pi)$, then as $k \rightarrow 1-0$

$$
h_{1}\left(v_{1}^{1}(k), k\right) \rightarrow \int_{0}^{\bar{v}}(|\cos t|-1 /|\cos t|) \mathrm{d} t-\sqrt{1-\sin ^{2} \bar{v}} \tan \bar{v}=\infty,
$$

which contradicts the identity $h_{1}\left(v_{1}^{1}(k), k\right) \equiv 0, k \in[0,1)$. Thus $\bar{v}=\pi / 2$.

(6) As $(k, v) \rightarrow(0, \pi)$, we have $h_{1}(v, k)=v-\pi+(\pi / 2) k^{2}+o\left(k^{2}+(v-\pi)^{2}\right)$, thus $v_{1}^{1}(k)=\pi-(\pi / 2) k^{2}+o\left(k^{2}\right)$, $k \rightarrow+0$.

A plot of the function $v_{1}^{1}(k)$ is given in Figure 7 .

Define the curve $\Gamma_{1} \subset S=\left\{q \in M \mid \theta \in(0, \pi), R_{1}>0, R_{2}=0\right\}$ given parametrically as follows:

$$
\begin{aligned}
& \theta=2 \arcsin \left(k \sin v_{1}^{1}(k)\right), \\
& R_{1}=2\left(F\left(v_{1}^{1}(k), k\right)-E\left(v_{1}^{1}(k), k\right)\right), \quad k \in[0,1),
\end{aligned}
$$

see Figure 8.

\section{Lemma 2.5.}

(1) The function $k \sin v_{1}^{1}(k)$ is strictly increasing as $k \in[0,1]$, thus the function $\theta=\theta(k), k \in[0,1]$, determined by (2.18) has an inverse function $k=k_{1}^{1}(\theta), \theta \in[0, \pi]$.

(2) $k_{1}^{1} \in C[0, \pi] \cap C^{\infty}[0, \pi)$.

(3) The function $k_{1}^{1}(\theta)$ is strictly increasing as $\theta \in[0, \pi]$.

(4) The curve $\Gamma_{1}$ is a graph of the function

$$
\begin{aligned}
& R_{1}=R_{1}^{1}(\theta), \quad \theta \in[0, \pi], \\
& R_{1}^{1}(\theta)=2\left(F\left(v_{1}^{1}(k), k\right)-E\left(v_{1}^{1}(k), k\right)\right), \quad k=k_{1}^{1}(\theta) .
\end{aligned}
$$

(5) $R_{1}^{1} \in C[0, \pi] \cap C^{\infty}(0, \pi)$.

(6) $R_{1}^{1}(\theta)=\sqrt[3]{\pi} / 2 \theta^{2 / 3}+o\left(\theta^{2 / 3}\right), \theta \rightarrow+0$.

Proof. (1) As $k \in[0,1]$, we have:

$$
\begin{aligned}
& v_{1}^{1}(k) \downarrow, \quad v_{1}^{1}(k) \in[\pi / 2, \pi], \\
& \sin v_{1}^{1}(k) \uparrow, \quad k \sin v_{1}^{1}(k) \uparrow, \quad 2 \arcsin \left(k v_{1}^{1}(k)\right) \uparrow .
\end{aligned}
$$


(2) follows from items (2) and (5) of Lemma 2.4.

(3) follows from item (1) of this lemma.

(4) follows from (2.18) and (2.19).

(5) follows from item (2) of this lemma.

(6) As $k \rightarrow+0$, we have

$$
v_{1}^{1}(k)=\pi-(\pi / 2) k^{2}+o\left(k^{2}\right), \quad \sin v_{1}^{1}(k)=(\pi / 2) k^{2}+o\left(k^{2}\right),
$$

and for the functions $(2.18),(2.19)$

$$
\theta=\pi k^{3}+o\left(k^{3}\right), \quad R_{1}=(\pi / 2) k^{2}+o\left(k^{2}\right) .
$$

Thus as $\theta \rightarrow+0$, we have

$$
k_{1}^{1}(\theta)=\sqrt[3]{\theta / \pi}+o(\sqrt[3]{\theta}), \quad R_{1}^{1}(\theta)=\sqrt[3]{\pi} / 2 \theta^{2 / 3}+o\left(\theta^{2 / 3}\right)
$$

Define the following subset of $M^{\prime}$, see Figure 14:

$$
M_{26}^{\prime}=\left\{q \in M \mid \theta \in(\pi, 2 \pi), R_{1}=R_{1}^{1}(2 \pi-\theta), R_{2}=0\right\} .
$$

Lemma 2.6. The mapping Exp : $N_{26}^{\prime} \rightarrow M_{26}^{\prime}$ is a diffeomorphism of 1-dimensional manifolds.

Proof. For $\nu \in N_{26}^{\prime}$ we obtain from formulas (5.7)-(5.12) [7]:

$$
\begin{aligned}
& \sin (\theta / 2)=k \operatorname{sn} p_{1}^{1}(k)=k \sin v_{1}^{1}(k), \\
& \cos (\theta / 2)=-\operatorname{dn} p_{1}^{1}(k)=-\sqrt{1-k^{2} \sin ^{2} v_{1}^{1}(k)}, \\
& R_{1}=2\left(p_{1}^{1}(k)-\mathrm{E}\left(p_{1}^{1}(k)\right)\right)=2\left(F\left(v_{1}^{1}(k), k\right)-E\left(v_{1}^{1}(k), k\right)\right), \\
& R_{2}=0 .
\end{aligned}
$$

Thus $\operatorname{Exp}\left(N_{26}^{\prime}\right)=M_{26}^{\prime}$. Moreover, the mapping $\operatorname{Exp}: N_{26}^{\prime} \rightarrow M_{26}^{\prime}$ decomposes into the chain

$$
\begin{aligned}
& N_{26}^{\prime} \stackrel{(*)}{\rightarrow} \Gamma_{1} \stackrel{(* *)}{\rightarrow} M_{26}^{\prime}, \\
& (*): k \mapsto\left(\theta=2 \arcsin \left(k \sin v_{1}^{1}(k)\right), R_{1}=2\left(F\left(v_{1}^{1}(k), k\right)-E\left(v_{1}^{1}(k), k\right)\right), R_{2}=0\right), \\
& (* *):\left(\theta, R_{1}, R_{2}\right) \mapsto\left(2 \pi-\theta, R_{1}, R_{2}\right) .
\end{aligned}
$$

The mapping $(*)$ is a diffeomorphism by Lemma 2.5. Thus Exp : $N_{26}^{\prime} \rightarrow M_{26}^{\prime}$ is a diffeomorphism.

2.2.4. Exponential mapping of the set $N_{52}^{\prime}$

\section{Lemma 2.7.}

(1) The functions $R_{1}^{1}(\theta), R_{1}^{2}(\theta)$ defined in (2.20) and (2.13) satisfy the inequality

$$
R_{1}^{2}(\theta)<R_{1}^{1}(\theta), \quad \theta \in(0, \pi) .
$$

(2) The mapping Exp : $N_{52}^{\prime} \rightarrow M_{52}^{\prime}$ is a diffeomorphism of 2-dimensional manifolds, where

$$
M_{52}^{\prime}=\left\{q \in M \mid \theta \in(\pi, 2 \pi), R_{1} \in\left(R_{1}^{2}(2 \pi-\theta), R_{1}^{1}(2 \pi-\theta)\right), R_{2}=0\right\},
$$

see Figure 14. 


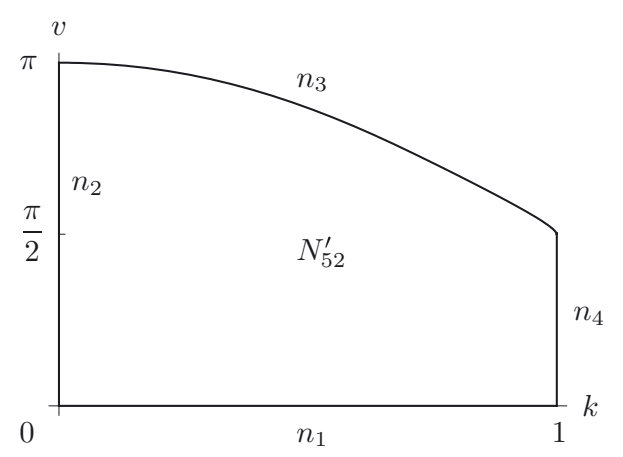

Figure 9. Domain $N_{52}^{\prime}$.

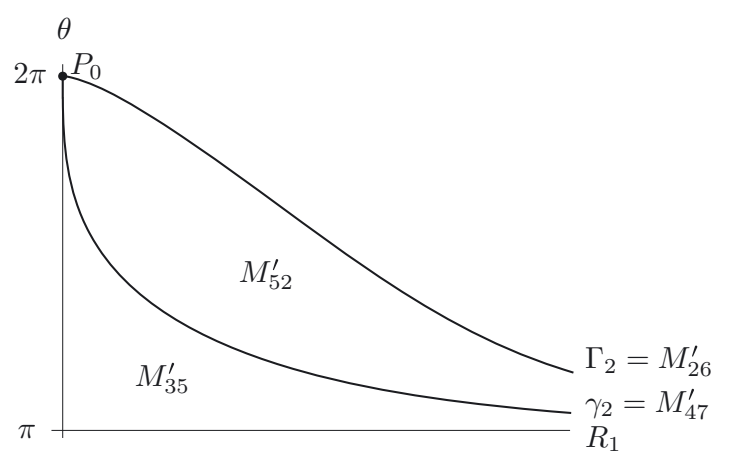

Figure 10. Curves $\gamma_{2}$ and $\Gamma_{2}$.

Proof. We have

where $v=\operatorname{am}(p, k)$. Thus

$$
N_{52}^{\prime}=\left\{\nu \in N_{2}^{+} \mid \tau=0, v \in\left(0, v_{1}^{1}(k)\right), k \in(0,1)\right\}
$$

$$
N_{52}^{\prime} \subset T=\left\{\nu \in N_{2}^{+} \mid \tau=0, v \in[0, \pi], k \in[0,1]\right\},
$$

and in the 2-dimensional topology of $T$

$$
\begin{aligned}
& \partial N_{52}^{\prime}=\cup_{i=1}^{4} n_{i}, \\
& n_{1}=\left\{\nu \in N_{2}^{+} \mid \tau=0, v=0, k \in[0,1]\right\}, \\
& n_{2}=\left\{\nu \in N_{2}^{+} \mid \tau=0, v \in[0, \pi], k=0\right\}, \\
& n_{3}=\left\{\nu \in N_{2}^{+} \mid \tau=0, v=v_{1}^{1}(k), k \in[0,1]\right\}, \\
& n_{4}=\left\{\nu \in N_{2}^{+} \mid \tau=0, v \in[0, \pi / 2], k=1\right\},
\end{aligned}
$$

see Figure 9.

By formulas (5.7)-(5.12) [7], the exponential mapping in the domain $N_{52}^{\prime}$ reads as follows:

$$
\begin{array}{ll}
\sin (\theta / 2)=k \sin v, & \cos (\theta / 2)=-\sqrt{1-k^{2} \sin ^{2} v}, \\
R_{1}=2(F(v, k)-E(v, k)), & R_{2}=0 .
\end{array}
$$

Thus

$$
\begin{aligned}
& \operatorname{Exp}\left(N_{52}^{\prime}\right) \subset S=\left\{q \in M \mid \theta \in(\pi, 2 \pi), R_{1}>0, R_{2}=0\right\}, \\
& \operatorname{Exp}\left(n_{1}\right)=\operatorname{Exp}\left(n_{2}\right)=P_{0}=\left\{q \in M \mid \theta=2 \pi, R_{1}=0, R_{2}=0\right\}, \\
& \operatorname{Exp}\left(n_{3}\right)=\overline{M_{26}^{\prime}}=\overline{\Gamma_{2}}, \quad \Gamma_{2}:=M_{26}^{\prime}, \\
& \operatorname{Exp}\left(n_{4}\right)=\overline{M_{47}^{\prime}}=\overline{\gamma_{2}}, \quad \gamma_{2}:=M_{47}^{\prime} .
\end{aligned}
$$

By Theorem 2.5 [10] (see (1.14)), the mapping $\left.\operatorname{Exp}\right|_{N_{52}^{\prime}}$ is nondegenerate, thus $\operatorname{Exp}\left(N_{52}^{\prime}\right)$ is an open connected domain in $S$, with $\partial \operatorname{Exp}\left(N_{52}^{\prime}\right) \subset \operatorname{Exp}\left(\partial N_{52}^{\prime}\right)=\overline{\Gamma_{2}} \cup \overline{\gamma_{2}}$.

The curves $\overline{\Gamma_{2}}$ and $\overline{\gamma_{2}}$ intersect one another at the point $P_{0}$. We show that they have no other intersection points. By contradiction, assume that the curves $\overline{\Gamma_{2}}$ and $\overline{\gamma_{2}}$ have intersection points distinct from $P_{0}$, then the domain $\operatorname{Exp}\left(N_{52}^{\prime}\right)$ is bounded by finite $\operatorname{arcs}$ of the curves $\overline{\Gamma_{2}}$ and $\overline{\gamma_{2}}$, i.e., there exists a point $P_{1} \in \gamma_{2} \cap \Gamma_{2}$, $P_{1} \neq P_{0}$, such that $\partial \operatorname{Exp}\left(N_{52}^{\prime}\right)=P_{0} \gamma_{2} P_{1} \cup P_{0} \Gamma_{2} P_{1}$. Then $\overline{\operatorname{Exp}\left(N_{52}^{\prime}\right)}$ does not contain the curves $\gamma_{2}, \Gamma_{2}$. 


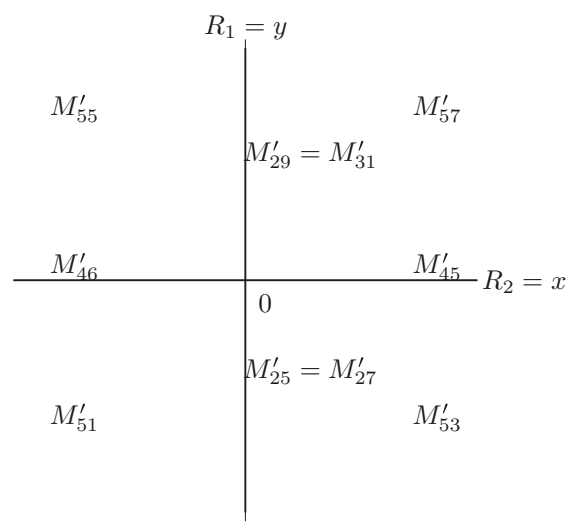

Figure 11. Decomposition of surface $\{\theta=0\}$.

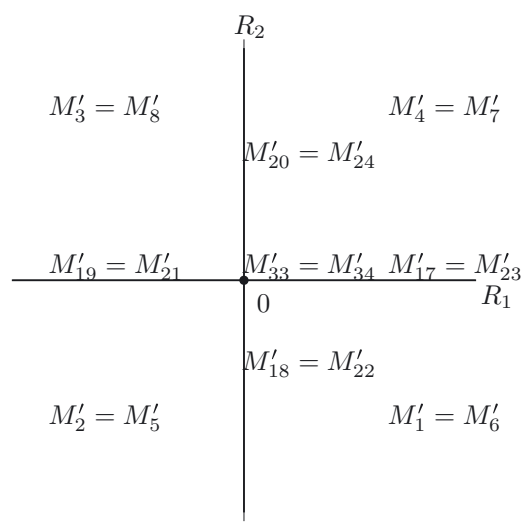

Figure 12. Decomposition of surface $\{\theta=\pi\}$.

This is a contradiction to the diffeomorphic property of the mapping Exp : $n_{3}=N_{26}^{\prime} \rightarrow \Gamma_{2}=M_{26}^{\prime}$ and Exp : $n_{4}=N_{47}^{\prime} \rightarrow \gamma_{2}=M_{47}^{\prime}$, see Lemmas 2.6 and 2.3 respectively.

Consequently, $\overline{\gamma_{2}} \cap \overline{\Gamma_{2}}=P_{0}$, and the domain $\operatorname{Exp}\left(N_{52}^{\prime}\right)$ is bounded by the curves $\overline{\gamma_{2}}, \overline{\Gamma_{2}}$.

The equalities $\frac{\mathrm{d} R_{1}^{1}}{\mathrm{~d} \theta}(0)=+\infty, \frac{\mathrm{d} R_{1}^{2}}{\mathrm{~d} \theta}(0)=0$ (see Lem. 2.5 and Eq. $(2.14)$ ) imply that $R_{1}^{2}(\theta)<R_{1}^{1}(\theta)$ for sufficiently small $\theta>0$. Further, the representations

$$
\begin{aligned}
& \Gamma_{2}=M_{26}^{\prime}=\left\{q \in M \mid \theta \in(\pi, 2 \pi), R_{1}=R_{1}^{1}(2 \pi-\theta), R_{2}=0\right\} \\
& \gamma_{2}=M_{47}^{\prime}=\left\{q \in M \mid \theta \in(\pi, 2 \pi), R_{1}=R_{1}^{2}(2 \pi-\theta), R_{2}=0\right\}
\end{aligned}
$$

imply the required inequality

$$
R_{1}^{2}(\theta)<R_{1}^{1}(\theta), \quad \theta \in(0, \pi)
$$

and the equality $\operatorname{Exp}\left(N_{52}^{\prime}\right)=M_{52}^{\prime}$

Since the mapping Exp : $N_{52}^{\prime} \rightarrow M_{52}^{\prime}$ is nondegenerate and proper, and the domains $N_{52}^{\prime}, M_{52}^{\prime}$ are open, connected, and simply connected, it follows that this mapping is a diffeomorphism.

The mutual disposition of the curves $\gamma_{2}=M_{47}^{\prime}$ and $\Gamma_{2}=M_{26}^{\prime}$ is shown in Figures 10 and 14 .

\subsection{Decomposition of the set $M^{\prime}$}

Now we have the functions $R_{1}^{2}(\theta)<R_{1}^{1}(\theta)$ required for definition of the following decomposition:

$$
M^{\prime}=\cup_{i=1}^{58} M_{i}^{\prime}
$$

where the subsets $M_{i}^{\prime}$ are defined by Table 2. Notice that some of the sets $M_{i}^{\prime}$ coincide between themselves, unlike the sets $N_{i}^{\prime}$, see (2.12). A precise definition of coinciding $M_{i}^{\prime}$ is given below in Theorem 3.1, item (1).

The structure of decomposition (2.21) in the surfaces $\{\theta=0\},\{\theta=\pi\},\left\{R_{1}=0\right\},\left\{R_{2}=0\right\}$ is shown respectively in Figures 11, 12, 13 and 14.

\subsection{Exponential mapping of the sets $N_{36}^{\prime}, N_{53}^{\prime}, N_{18}^{\prime}, N_{33}^{\prime}, N_{34}^{\prime}, N_{17}^{\prime}, N_{27}^{\prime}, N_{1}^{\prime}, N_{10}^{\prime}$}

2.4.1. Exponential mapping of the set $N_{36}^{\prime}$

Lemma 2.8. The mapping Exp : $N_{36}^{\prime} \rightarrow M_{36}^{\prime}$ is a diffeomorphism of 2-dimensional manifolds. 


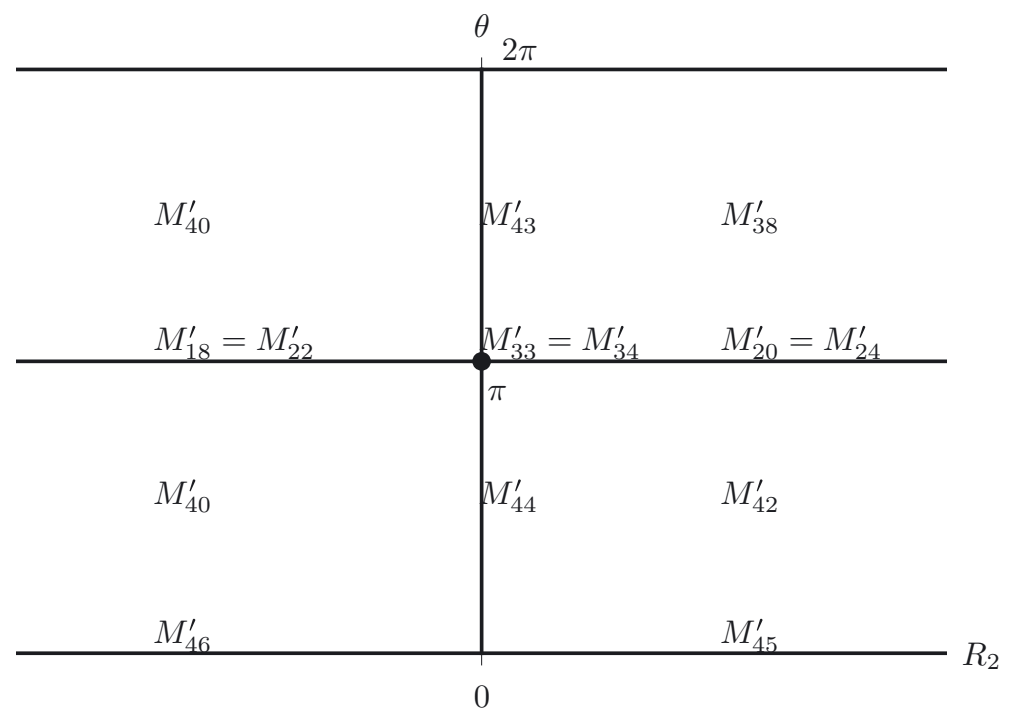

Figure 13. Decomposition of surface $\left\{R_{1}=0\right\}$.

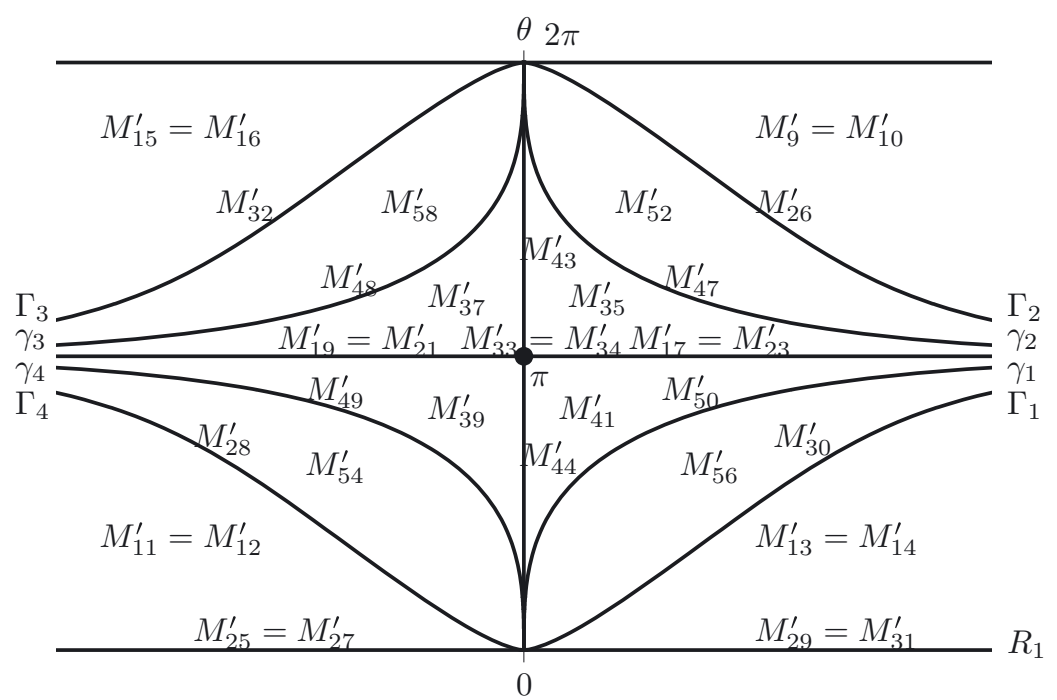

Figure 14. Decomposition of surface $\left\{R_{2}=0\right\}$.

Proof. By formulas (5.2)-(5.6) [7], exponential mapping in the domain $N_{36}^{\prime}$ reads as follows:

$$
\begin{array}{ll}
\sin (\theta / 2)=\sqrt{1-k^{2}} \operatorname{sn} p / \operatorname{dn} p, & \cos (\theta / 2)=-\operatorname{cn} p / \operatorname{dn} p \\
R_{1}=0, & R_{2}=-2 f_{2}(p, k) /(k \operatorname{dn} p),
\end{array}
$$

where $f_{2}(p, k)=k^{2} \operatorname{cn} p \operatorname{sn} p+\operatorname{dn} p(p-\mathrm{E}(p))>0$ by Lemma 5.2 [7], thus $\operatorname{Exp}\left(N_{36}^{\prime}\right) \subset M_{36}^{\prime}$. 
TABle 2. Definition of sets $M_{i}^{\prime}$.

\begin{tabular}{|c|c|c|c|c|c|c|}
\hline$M_{i}^{\prime}$ & $M_{1}^{\prime}=M_{6}^{\prime}$ & $M_{2}^{\prime}=M_{5}^{\prime}$ & $M_{3}^{\prime}=M_{8}^{\prime}$ & $M_{4}^{\prime}=M_{7}^{\prime}$ & $M_{9}^{\prime}=M_{10}^{\prime}$ & $M_{11}^{\prime}=M_{12}^{\prime}$ \\
\hline$\theta$ & $\pi$ & $\pi$ & $\pi$ & $\pi$ & $(\pi, 2 \pi)$ & $(0, \pi)$ \\
\hline$R_{1}$ & $(0,+\infty)$ & $(-\infty, 0)$ & $(-\infty, 0)$ & $(0,+\infty)$ & $\left(R_{1}^{1}(2 \pi-\theta),+\infty\right)$ & $\left(-\infty,-R_{1}^{1}(\theta)\right)$ \\
\hline$R_{2}$ & $(-\infty, 0)$ & $(-\infty, 0)$ & $(0,+\infty)$ & $(0,+\infty)$ & 0 & 0 \\
\hline
\end{tabular}

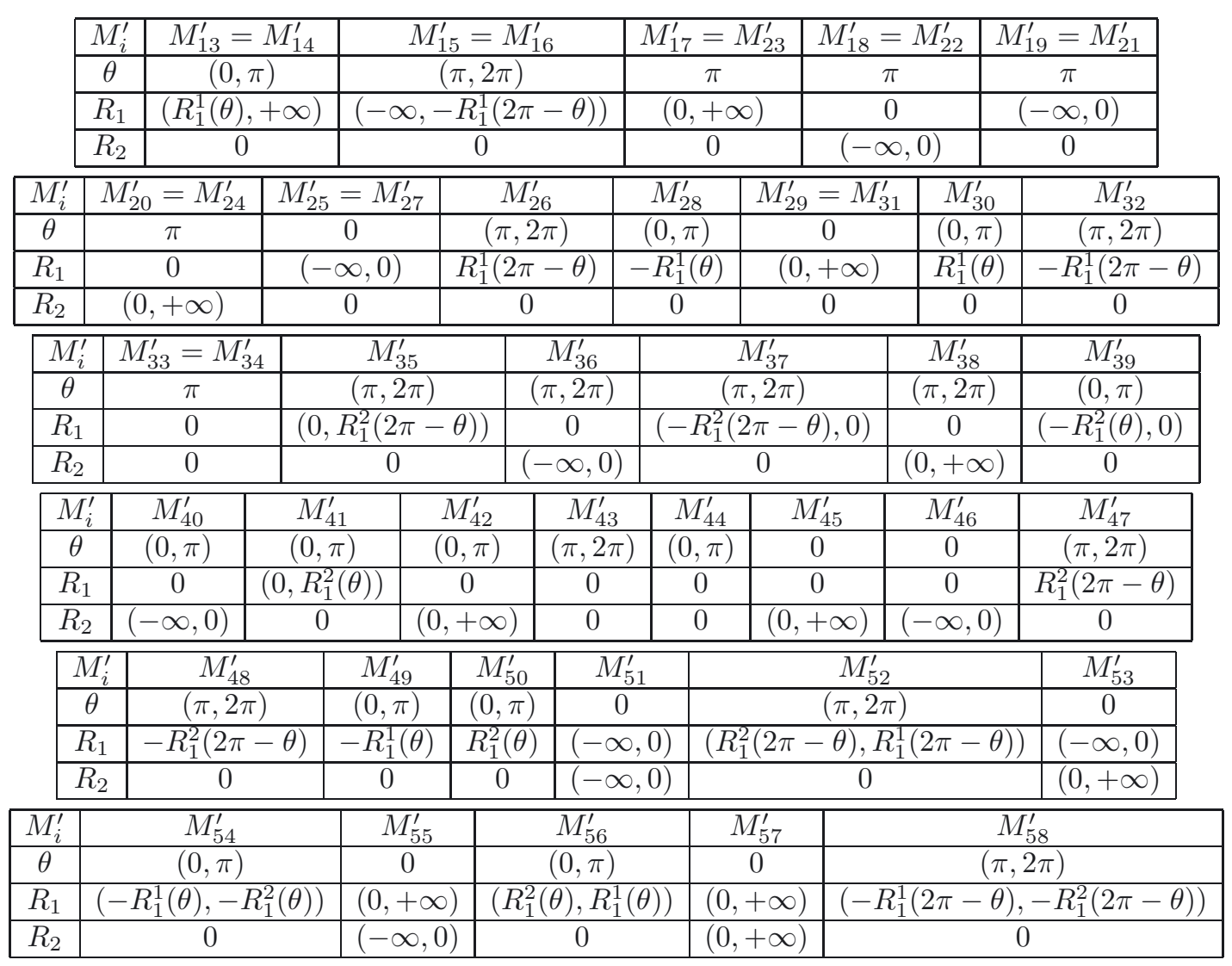

In the topology of the manifold $\left\{R_{1}=0\right\}$, we have:

$$
\begin{aligned}
& \partial N_{36}^{\prime}=\cup_{i=1}^{4} n_{i}, \\
& n_{1}=\left\{\nu \in N_{1}^{0} \mid \tau=K, p=0, k \in[0,1]\right\}, \\
& n_{2}=\left\{\nu \in N_{1}^{0} \mid \tau=K, p \in[0, \pi / 2], k=0\right\}, \\
& n_{3}=\left\{\nu \in N_{1}^{0} \mid \tau=K, p=K, k \in[0,1)\right\}, \\
& n_{4}=\left\{\nu \in N_{1}^{0} \mid \tau=K, p \in[0,+\infty), k=1\right\},
\end{aligned}
$$

see Figure 15. 


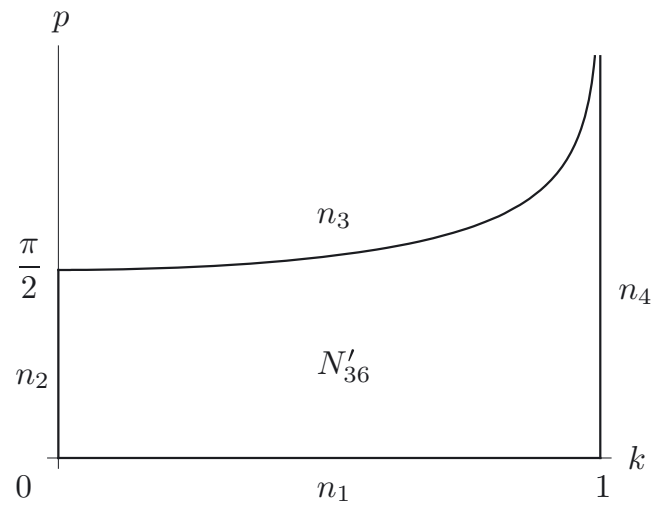

Figure 15. Domain $N_{36}^{\prime}$.

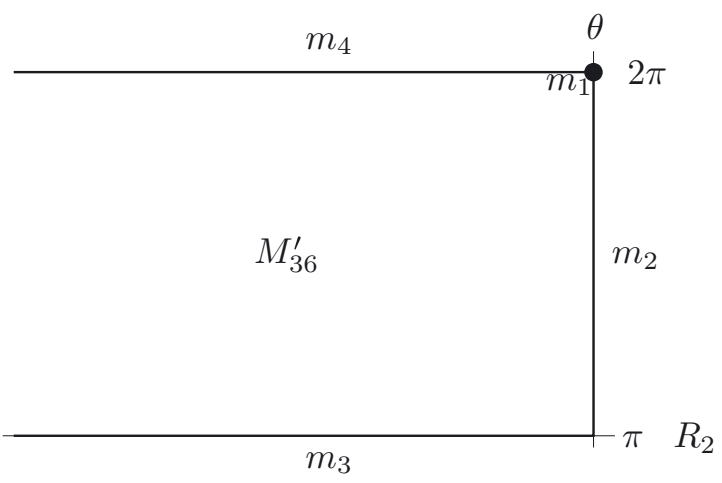

Figure 16. Domain $M_{36}^{\prime}$.

Further, we have $\operatorname{Exp}\left(n_{i}\right)=m_{i}, i=1, \ldots, 4$, where

$$
\begin{aligned}
& m_{1}=\left\{q \in M \mid \theta=2 \pi, R_{1}=0, R_{2}=0\right\}, \\
& m_{2}=\left\{q \in M \mid \theta \in[\pi, 2 \pi], R_{1}=0, R_{2}=0\right\}, \\
& m_{3}=\left\{q \in M \mid \theta=\pi, R_{1}=0, R_{2} \in(-\infty, 0]\right\}, \\
& m_{4}=\left\{q \in M \mid \theta=2 \pi, R_{1}=0, R_{2} \in(-\infty, 0]\right\},
\end{aligned}
$$

see Figure 16.

The mapping Exp : $N_{36}^{\prime} \rightarrow M_{36}^{\prime}$ is nondegenerate and proper, the domains $N_{36}^{\prime}, M_{36}^{\prime}$ are open (in the 2-dimensional topology), connected and simply connected, thus it is a diffeomorphism.

\subsubsection{Exponential mapping of the set $N_{53}^{\prime}$}

Lemma 2.9. The mapping Exp : $N_{53}^{\prime} \rightarrow M_{53}^{\prime}$ is a diffeomorphism of 2-dimensional manifolds.

Proof. The argument follows similarly to the proof of Lemma 2.8 via the following coordinate representation of the exponential mapping in the domain $N_{53}^{\prime}$ :

$$
\theta=0, \quad R_{1}=-2 \sqrt{1-k^{2}}(p-\mathrm{E}(p)) / \operatorname{dn} p<0, \quad R_{2}=-2 k f_{1}(p, k) / \operatorname{dn} p,
$$

where $f_{1}(p, k)=\operatorname{cn} p(\mathrm{E}(p)-p)-\operatorname{dn} p \operatorname{sn} p<0$ for $p \in\left(0, p_{1}^{1}\right)$, see Corollary 5.1 [7].

2.4.3. Exponential mapping of the set $N_{18}^{\prime}$

Lemma 2.10. The mapping Exp $: N_{18}^{\prime} \rightarrow M_{18}^{\prime}$ is a diffeomorphism of 1-dimensional manifolds.

Proof. By formulas (5.2)-(5.6) [7], we have in the set $N_{18}^{\prime}$ :

$$
\theta=\pi, \quad R_{1}=0, \quad R_{2}=-(2 / k)(K(k)-E(k)),
$$

and the diffeomorphic property of $\left.\operatorname{Exp}\right|_{N_{18}^{\prime}}$ follows as usual from its nondegeneracy and properness, and topological properties of the sets $N_{18}^{\prime}, M_{18}^{\prime}$.

2.4.4. Exponential mapping of the sets $N_{33}^{\prime}, N_{34}^{\prime}$

Lemma 2.11. The mappings Exp : $N_{33}^{\prime} \rightarrow M_{33}^{\prime}$ and $\operatorname{Exp}: N_{34}^{\prime} \rightarrow M_{34}^{\prime}$ are diffeomorphisms (bijections) of o-dimensional manifolds.

Proof. Obvious. 
2.4.5. Exponential mapping of the set $N_{17}^{\prime}$

Lemma 2.12. The mapping Exp : $N_{17}^{\prime} \rightarrow M_{17}^{\prime}$ is a diffeomorphism of 1-dimensional manifolds.

Proof. The statement follows as in the proof of Lemma 2.10 via the following coordinate representation of the exponential mapping in the domain $N_{17}^{\prime}$ :

$$
\theta=\pi, \quad R_{1}=(2 / k)(K(k)-E(k)), \quad R_{2}=0 .
$$

2.4.6. Exponential mapping of the set $N_{27}^{\prime}$

Lemma 2.13. The mapping Exp : $N_{27}^{\prime} \rightarrow M_{27}^{\prime}$ is a diffeomorphism of 2-dimensional manifolds.

Proof. Formulas (5.7)-(5.12) [7] yield:

$$
\theta=\pi, \quad R_{1}=-2 \sqrt{1-k^{2}}(p-\mathrm{E}(p)) /\left.\operatorname{dn} p\right|_{p=p_{1}^{1}(k)}, \quad R_{2}=0 .
$$

Since $\tau=K$, then Lemma 2.4 [10] implies that the mapping $\left.\operatorname{Exp}\right|_{N_{27}^{\prime}}$ is nondegenerate. Then the diffeomorphic property of Exp : $N_{27}^{\prime} \rightarrow M_{27}^{\prime}$ follows as usual.

2.4.7. Exponential mapping of the set $N_{1}^{\prime}$

Lemma 2.14. The mapping Exp : $N_{1}^{\prime} \rightarrow M_{1}^{\prime}$ is a diffeomorphism of 2-dimensional manifolds.

Proof. Formulas (5.2)-(5.6) [7] yield:

$$
\begin{aligned}
& \theta=\pi, \quad R_{1}=2(K(k)-E(k)) \operatorname{cn} \tau /(k \operatorname{dn} \tau)>0, \\
& R_{2}=-2 \sqrt{1-k^{2}}(K(k)-E(k)) \operatorname{sn} \tau /(k \operatorname{dn} \tau)<0,
\end{aligned}
$$

and the statement follows as usual since the mapping $\left.\operatorname{Exp}\right|_{N_{1}^{\prime}}$ is nondegenerate and proper.

2.4.8. Exponential mapping of the set $N_{10}^{\prime}$

Lemma 2.15. The mapping Exp : $N_{10}^{\prime} \rightarrow M_{10}^{\prime}$ is a diffeomorphism of 2-dimensional manifolds.

Proof. By formulas (5.7)-(5.12) [7] we get:

$$
\begin{array}{ll}
\sin (\theta / 2)=k \operatorname{sn} p_{1}^{1} \operatorname{cn} \tau / \sqrt{\Delta}>0, & \cos (\theta / 2)=-\operatorname{dn} p_{1}^{1} / \sqrt{\Delta}<0, \\
R_{1}=2(p-\mathrm{E}(p)) \operatorname{dn} \tau /\left.\sqrt{\Delta}\right|_{p=p_{1}^{1}}>0, & R_{2}=0,
\end{array}
$$

where $\Delta=1-k^{2} \operatorname{sn}^{2} p \operatorname{sn}^{2} \tau$, and the statement follows by standard argument since $\left.\operatorname{Exp}\right|_{N_{10}^{\prime}}$ is nondegenerate and proper.

\subsection{Action of the group of reflections in the preimage and image of the exponential mapping}

In order to extend the results of the preceding subsections to all 58 pairs $\left(N_{i}^{\prime}, M_{i}^{\prime}\right), i \in I$, we describe the action of the group of reflections $G=\left\{\operatorname{Id}, \varepsilon^{1}, \ldots, \varepsilon^{7}\right\}$ on these sets.

Theorem 2.1. Tables 3, 4, 5, 9 and 6, 7, 8, 10 define diffeomorphisms between the corresponding manifolds $N_{i}^{\prime}$ and $M_{i}^{\prime}$.

Proof. Follows from definitions of the manifolds $N_{i}^{\prime}$ and $M_{i}^{\prime}$ (Sects. 2.1 and 2.3) and Propositions 4.3 and 4.4 [7] describing action of the reflections $\varepsilon^{i} \in G$ in the image and preimage of the exponential mapping. Moreover, in the coordinates $\left(\theta, R_{1}, R_{2}\right)$ action of the reflections is described by Table 11 . 
TABle 3. Action of $\varepsilon^{1}, \varepsilon^{4}, \varepsilon^{5}$ on $N_{35}^{\prime}, N_{47}^{\prime}, N_{52}^{\prime}, N_{17}^{\prime}, N_{26}^{\prime}$.

\begin{tabular}{|c|l|l|l|l|l|}
\hline$D$ & $N_{35}^{\prime}$ & $N_{47}^{\prime}$ & $N_{52}^{\prime}$ & $N_{17}^{\prime}$ & $N_{26}^{\prime}$ \\
\hline$\varepsilon^{1}(D)$ & $N_{37}^{\prime}$ & $N_{48}^{\prime}$ & $N_{58}^{\prime}$ & $N_{19}^{\prime}$ & $N_{32}^{\prime}$ \\
\hline$\varepsilon^{4}(D)$ & $N_{39}^{\prime}$ & $N_{49}^{\prime}$ & $N_{54}^{\prime}$ & $N_{21}^{\prime}$ & $N_{28}^{\prime}$ \\
\hline$\varepsilon^{5}(D)$ & $N_{41}^{\prime}$ & $N_{50}^{\prime}$ & $N_{56}^{\prime}$ & $N_{23}^{\prime}$ & $N_{30}^{\prime}$ \\
\hline
\end{tabular}

TABLE 4. Action of $\varepsilon^{2}, \varepsilon^{4}, \varepsilon^{6}$ on $N_{36}^{\prime}, N_{18}^{\prime}$.

\begin{tabular}{|c|l|l|}
\hline$D$ & $N_{36}^{\prime}$ & $N_{18}^{\prime}$ \\
\hline$\varepsilon^{2}(D)$ & $N_{38}^{\prime}$ & $N_{20}^{\prime}$ \\
\hline$\varepsilon^{4}(D)$ & $N_{40}^{\prime}$ & $N_{22}^{\prime}$ \\
\hline$\varepsilon^{6}(D)$ & $N_{42}^{\prime}$ & $N_{24}^{\prime}$ \\
\hline
\end{tabular}

TABle 5. Action of $\varepsilon^{1}, \varepsilon^{2}, \varepsilon^{3}$ on $N_{53}^{\prime}, N_{27}^{\prime}$.

\begin{tabular}{|c|l|l|}
\hline$D$ & $N_{53}^{\prime}$ & $N_{27}^{\prime}$ \\
\hline$\varepsilon^{1}(D)$ & $N_{57}^{\prime}$ & $N_{31}^{\prime}$ \\
\hline$\varepsilon^{2}(D)$ & $N_{51}^{\prime}$ & $N_{25}^{\prime}$ \\
\hline$\varepsilon^{3}(D)$ & $N_{55}^{\prime}$ & $N_{29}^{\prime}$ \\
\hline
\end{tabular}

TABle 6. Action of $\varepsilon^{1}, \varepsilon^{4}, \varepsilon^{5}$ on $M_{35}^{\prime}, M_{47}^{\prime}, M_{52}^{\prime}, M_{17}^{\prime}, M_{26}^{\prime}$.

\begin{tabular}{|c|l|l|l|l|l|}
\hline$D$ & $M_{35}^{\prime}$ & $M_{47}^{\prime}$ & $M_{52}^{\prime}$ & $M_{17}^{\prime}$ & $M_{26}^{\prime}$ \\
\hline$\varepsilon^{1}(D)$ & $M_{37}^{\prime}$ & $M_{48}^{\prime}$ & $M_{58}^{\prime}$ & $M_{19}^{\prime}$ & $M_{32}^{\prime}$ \\
\hline$\varepsilon^{4}(D)$ & $M_{39}^{\prime}$ & $M_{49}^{\prime}$ & $M_{54}^{\prime}$ & $M_{21}^{\prime}$ & $M_{28}^{\prime}$ \\
\hline$\varepsilon^{5}(D)$ & $M_{41}^{\prime}$ & $M_{50}^{\prime}$ & $M_{56}^{\prime}$ & $M_{23}^{\prime}$ & $M_{30}^{\prime}$ \\
\hline
\end{tabular}

TABle 7. Action of $\varepsilon^{2}, \varepsilon^{4}, \varepsilon^{6}$ on $M_{36}^{\prime}, M_{18}^{\prime}$.

\begin{tabular}{|c|l|l|}
\hline$D$ & $M_{36}^{\prime}$ & $M_{18}^{\prime}$ \\
\hline$\varepsilon^{2}(D)$ & $M_{38}^{\prime}$ & $M_{20}^{\prime}$ \\
\hline$\varepsilon^{4}(D)$ & $M_{40}^{\prime}$ & $M_{22}^{\prime}$ \\
\hline$\varepsilon^{6}(D)$ & $M_{42}^{\prime}$ & $M_{24}^{\prime}$ \\
\hline
\end{tabular}

TABle 8. Action of $\varepsilon^{1}, \varepsilon^{2}, \varepsilon^{3}$ on $M_{53}^{\prime}, M_{27}^{\prime}$.

\begin{tabular}{|c|l|l|}
\hline$D$ & $M_{53}^{\prime}$ & $M_{27}^{\prime}$ \\
\hline$\varepsilon^{1}(D)$ & $M_{57}^{\prime}$ & $M_{31}^{\prime}$ \\
\hline$\varepsilon^{2}(D)$ & $M_{51}^{\prime}$ & $M_{25}^{\prime}$ \\
\hline$\varepsilon^{3}(D)$ & $M_{55}^{\prime}$ & $M_{29}^{\prime}$ \\
\hline
\end{tabular}

\subsection{The final result for exponential mapping of the sets $N_{i}^{\prime}$}

Theorem 2.2. For any $i \in I$, the mapping Exp : $N_{i}^{\prime} \rightarrow M_{i}^{\prime}$ is a diffeomorphism of manifolds of appropriate dimension 2, 1, or 0 .

Proof. For $i \in\{35,47,26,52,36,53,18,17,27,1,10\}$ the statement follows from Lemmas 2.2, 2.3, 2.6, 2.7, 2.8, $2.9,2.10,2.12,2.13,2.14$ and 2.15 respectively.

For $i \in\{33,34\}$ the statement was proved in Lemma 2.11.

For all the rest $i$ the statement follows from the above lemmas and Theorem 2.1 since the reflections $\varepsilon^{i} \in G$ are symmetries of the exponential mapping, see Proposition 4.5 [7].

\subsection{Reflections $\varepsilon^{k}$ as permutations}

In addition to the index sets $I, C, J, R, X$ introduced in (2.9) and (2.10), we will need also the set

$$
T=\left\{(i, j) \in I \times I \mid i<j, M_{i}^{\prime}=M_{j}^{\prime}\right\} .
$$


TABLE 9. Action of $\varepsilon^{1}, \ldots, \varepsilon^{7}$ on $N_{1}^{\prime}, N_{10}^{\prime}$.

\begin{tabular}{|c|c|c|}
\hline$D$ & $N_{1}^{\prime}$ & $N_{10}^{\prime}$ \\
\hline$\varepsilon^{1}(D)$ & $N_{2}^{\prime}$ & $N_{15}^{\prime}$ \\
\hline$\varepsilon^{2}(D)$ & $N_{4}^{\prime}$ & $N_{9}^{\prime}$ \\
\hline$\varepsilon^{3}(D)$ & $N_{3}^{\prime}$ & $N_{16}^{\prime}$ \\
\hline$\varepsilon^{4}(D)$ & $N_{5}^{\prime}$ & $N_{12}^{\prime}$ \\
\hline$\varepsilon^{5}(D)$ & $N_{6}^{\prime}$ & $N_{13}^{\prime}$ \\
\hline$\varepsilon^{6}(D)$ & $N_{8}^{\prime}$ & $N_{11}^{\prime}$ \\
\hline$\varepsilon^{7}(D)$ & $N_{7}^{\prime}$ & $N_{14}^{\prime}$ \\
\hline
\end{tabular}

TABLE 10. Action of $\varepsilon^{1}, \ldots, \varepsilon^{7}$ on $M_{1}^{\prime}, M_{10}^{\prime}$.

\begin{tabular}{|c|c|c|}
\hline$D$ & $M_{1}^{\prime}$ & $M_{10}^{\prime}$ \\
\hline$\varepsilon^{1}(D)$ & $M_{2}^{\prime}$ & $M_{15}^{\prime}$ \\
\hline$\varepsilon^{2}(D)$ & $M_{4}^{\prime}$ & $M_{9}^{\prime}$ \\
\hline$\varepsilon^{3}(D)$ & $M_{3}^{\prime}$ & $M_{16}^{\prime}$ \\
\hline$\varepsilon^{4}(D)$ & $M_{5}^{\prime}$ & $M_{12}^{\prime}$ \\
\hline$\varepsilon^{5}(D)$ & $M_{6}^{\prime}$ & $M_{13}^{\prime}$ \\
\hline$\varepsilon^{6}(D)$ & $M_{8}^{\prime}$ & $M_{11}^{\prime}$ \\
\hline$\varepsilon^{7}(D)$ & $M_{7}^{\prime}$ & $M_{14}^{\prime}$ \\
\hline
\end{tabular}

TABLE 11. Action of $\varepsilon^{1}, \ldots, \varepsilon^{7}$ on $M=\left\{\left(R_{1}, R_{2}, \theta\right)\right\}$.

\begin{tabular}{|c|c|c|c|c|c|c|c|}
\hline & $\varepsilon^{1}$ & $\varepsilon^{2}$ & $\varepsilon^{3}$ & $\varepsilon^{4}$ & $\varepsilon^{5}$ & $\varepsilon^{6}$ & $\varepsilon^{7}$ \\
\hline$R_{1}$ & $-R_{1}$ & $R_{1}$ & $-R_{1}$ & $-R_{1}$ & $R_{1}$ & $-R_{1}$ & $R_{1}$ \\
\hline$R_{2}$ & $R_{2}$ & $-R_{2}$ & $-R_{2}$ & $R_{2}$ & $R_{2}$ & $-R_{2}$ & $-R_{2}$ \\
\hline$\theta$ & $\theta$ & $\theta$ & $\theta$ & $2 \pi-\theta$ & $2 \pi-\theta$ & $2 \pi-\theta$ & $2 \pi-\theta$ \\
\hline
\end{tabular}

TABLE 12. Multiplication table in the group $G$.

\begin{tabular}{|l|l|l|l|l|l|l|l|}
\hline & $\varepsilon^{1}$ & $\varepsilon^{2}$ & $\varepsilon^{3}$ & $\varepsilon^{4}$ & $\varepsilon^{5}$ & $\varepsilon^{6}$ & $\varepsilon^{7}$ \\
\hline$\varepsilon^{1}$ & $\mathrm{Id}$ & $\varepsilon^{3}$ & $\varepsilon^{2}$ & $\varepsilon^{5}$ & $\varepsilon^{4}$ & $\varepsilon^{7}$ & $\varepsilon^{6}$ \\
\hline$\varepsilon^{2}$ & & $\mathrm{Id}$ & $\varepsilon^{1}$ & $\varepsilon^{6}$ & $\varepsilon^{7}$ & $\varepsilon^{4}$ & $\varepsilon^{5}$ \\
\hline$\varepsilon^{3}$ & & & $\mathrm{Id}$ & $\varepsilon^{7}$ & $\varepsilon^{6}$ & $\varepsilon^{5}$ & $\varepsilon^{4}$ \\
\hline$\varepsilon^{4}$ & & & & $\mathrm{Id}$ & $\varepsilon^{1}$ & $\varepsilon^{2}$ & $\varepsilon^{3}$ \\
\hline$\varepsilon^{5}$ & & & & & $\mathrm{Id}$ & $\varepsilon^{3}$ & $\varepsilon^{2}$ \\
\hline$\varepsilon^{6}$ & & & & & & $\mathrm{Id}$ & $\varepsilon^{1}$ \\
\hline$\varepsilon^{7}$ & & & & & & & $\mathrm{Id}$ \\
\hline
\end{tabular}

From the definition of the sets $M_{i}^{\prime}$ in Section 2.3 we obtain the explicit representation:

$T=\{(1,6),(2,5),(3,8),(4,7),(9,10),(11,12),(13,14),(15,16)$,

$(17,23),(18,22),(19,21),(20,24),(25,27),(29,31),(33,34)\}$.

Notice that $X=\{i \in I \mid \exists j \in I:(i, j) \in T$ or $(j, i) \in T\}$.

Now we show that reflections $\varepsilon^{k} \in G$ permute elements in any pair $(i, j) \in T$.

We will need multiplication Table 12 in the group $G$, which follows from definitions of the reflections $\varepsilon^{k}$ (Sect. 4 [7]). The lower diagonal entries of the table are not filled since $G$ is Abelian.

Lemma 2.16. For any $(i, j) \in T$ there exists a reflection $\varepsilon^{k} \in G$ such that the following diagram is commutative:
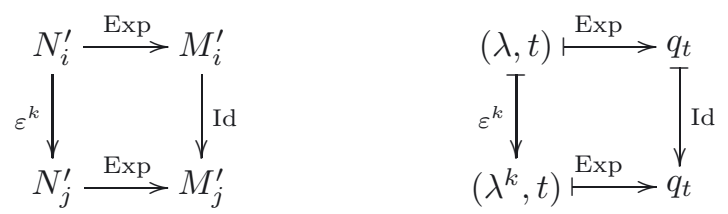
Proof. From definitions of the sets $N_{i}^{\prime}$ (Sect. 2.1) and the reflections $\varepsilon^{k}$ (Sect. 4 [7]), Tables 3, 4, 5, 9, 12 and Proposition 4.5 [7], we obtain the following indices $k$ of required symmetries $\varepsilon^{k}$ for pairs $(i, j) \in T$ :

$$
\begin{aligned}
& (i, j) \in\{(1,6),(2,5),(3,8),(4,7),(17,23),(19,21)\} \Rightarrow k=5, \\
& (i, j) \in\{(9,10),(15,16),(11,12),(13,14),(25,27),(29,31)\} \Rightarrow k=2, \\
& (i, j) \in\{(33,34),(18,22),(20,24)\} \Rightarrow k=4 .
\end{aligned}
$$

\section{Solution to optimal CONTROL PROBlem}

In this section we present the final results of this study of the sub-Riemannian problem on $\mathrm{SE}(2)$.

\subsection{Global structure of the exponential mapping}

We say that a mapping $F: X \rightarrow Y$ is double if any point $y \in Y$ has exactly two preimages:

$$
\forall y \in Y \quad F^{-1}(y)=\left\{x_{1}, x_{2}\right\}, \quad x_{1} \neq x_{2} .
$$

\section{Theorem 3.1.}

(1) There is the following decomposition of preimage of the exponential mapping $\operatorname{Exp}: \widehat{N} \rightarrow \widehat{M}$ :

$$
\begin{aligned}
& \widehat{N}=\widetilde{N} \sqcup N^{\prime}, \\
& \widetilde{N}=\sqcup_{i=1}^{8} D_{i}, \\
& N^{\prime}=N_{\text {Max }} \sqcup N_{\text {conj }} \sqcup N_{\text {rest }}, \\
& N_{\text {Max }}=\sqcup_{i \in X} N_{i}^{\prime}, \\
& N_{\text {conj }}=\sqcup_{i \in J} N_{i}^{\prime}, \\
& N_{\text {rest }}=\sqcup_{i \in R} N_{i}^{\prime},
\end{aligned}
$$

and in the image of the exponential mapping:

$$
\begin{aligned}
& \widehat{M}=\widetilde{M} \sqcup M^{\prime}, \\
& \widetilde{M}=\sqcup_{i=1}^{8} M_{i}, \\
& M^{\prime}=M_{\text {Max }} \sqcup M_{\text {conj }} \sqcup M_{\text {rest }}, \\
& M_{\text {Max }}=\cup_{i \in X} M_{i}^{\prime}, \\
& M_{i}^{\prime} \cap M_{j}^{\prime} \neq \emptyset, i<j \quad \Rightarrow \quad(i, j) \in T, \quad\{i, j\} \subset X, \\
& (i, j) \in T \quad \Rightarrow \quad M_{i}^{\prime}=M_{j}^{\prime}, \\
& M_{\text {conj }}=\sqcup_{i \in J} M_{i}^{\prime}, \\
& M_{\text {rest }}=\sqcup_{i \in R} M_{i}^{\prime} .
\end{aligned}
$$

(2) In terms of these decompositions the exponential mapping $\operatorname{Exp}: \widehat{N} \rightarrow \widehat{M}$ has the following structure:

$$
\begin{aligned}
& \operatorname{Exp}: D_{i} \rightarrow M_{i} \text { is a diffeomorphism } \forall i=1, \ldots, 8, \\
& \operatorname{Exp}: N_{i}^{\prime} \rightarrow M_{i}^{\prime} \text { is a diffeomorphism } \forall i \in I .
\end{aligned}
$$


Thus

$$
\begin{aligned}
& \operatorname{Exp}: \widetilde{N} \rightarrow \widetilde{M} \text { is a bijection, } \\
& \operatorname{Exp}: N_{\mathrm{Max}} \rightarrow M_{\mathrm{Max}} \text { is a double mapping, } \\
& \operatorname{Exp}: N_{\mathrm{conj}} \rightarrow M_{\mathrm{conj}} \text { is a bijection, } \\
& \operatorname{Exp}: N_{\text {rest }} \rightarrow M_{\text {rest }} \text { is a bijection. }
\end{aligned}
$$

(3) Any point $q \in \widetilde{M}\left(q \in M_{\text {conj }}, q \in M_{\text {rest }}\right)$ has a unique preimage $\nu=\operatorname{Exp}^{-1}(q)$ for the mapping $\left.\operatorname{Exp}\right|_{\widehat{N}}: \widehat{N} \rightarrow \widehat{M}$. Moreover, $\nu \in \widetilde{N}$ (resp., $\nu \in N_{\text {conj }}, \nu \in N_{\text {rest }}$ ).

(4) Any point $q \in M_{\text {Max }}$ has exactly two preimages $\left\{\nu^{\prime}, \nu^{\prime \prime}\right\}=\operatorname{Exp}^{-1}(q)$ for the mapping $\left.\operatorname{Exp}\right|_{\widehat{N}}: \widehat{N} \rightarrow \widehat{M}$. Moreover, $\nu^{\prime}, \nu^{\prime \prime} \in N_{\text {Max }}$ and $\nu^{\prime \prime}=\varepsilon^{k}\left(\nu^{\prime}\right)$ for some $\varepsilon^{k} \in G$.

The domains $D_{i}$ and $M_{i}$ were defined in Tables 1 and 2 [10].

Proof. Equalities in item (1) follow immediately from definitions of the corresponding decompositions.

(2) Property (3.1) was proved in Theorem 3.1 [10], and property (3.3) is its corollary, with account of item (1). Property (3.2) was proved in Theorem 3.2, and properties (3.4)-(3.6) are its corollaries, with account of item (1).

(3) The statement follows from (3.3), (3.4), (3.5), (3.6), with account of item (1).

(4) The statement follows from (3.4), (3.5), (3.6), and Lemma 2.16.

\subsection{Optimal synthesis}

Theorem 3.2. Let $q \in \widehat{M}=M \backslash\left\{q_{0}\right\}$.

(1) Let $q \in \widetilde{M} \cup M_{\text {conj }} \cup M_{\text {rest }}=\widehat{M} \backslash M_{\text {Max }}$. Denote $\nu=(\lambda, t)=\operatorname{Exp}^{-1}(q) \in \widetilde{N} \cup N_{\text {conj }} \cup N_{\text {rest }}=\widehat{N} \backslash N_{\text {Max }}$. Then $q_{s}=\operatorname{Exp}(\lambda, s), s \in[0, t]$, is the unique optimal trajectory connecting $q_{0}$ with $q$. If $q \in \widetilde{M} \cup M_{\text {rest }}$, then $t<\mathbf{t}(\lambda)$; if $q \in M_{\text {conj }}$, then $t=\mathbf{t}(\lambda)=t_{1}^{\operatorname{conj}}(\lambda)$.

(2) Let $q \in M_{\text {Max }}$. Denote $\left\{\nu^{\prime}, \nu^{\prime \prime}\right\}=\operatorname{Exp}^{-1}(q) \subset N_{\text {Max }}, \nu^{\prime}=\left(\lambda^{\prime}, t\right) \neq \nu^{\prime \prime}=\left(\lambda^{\prime \prime}, t\right)$. Then there exist exactly two distinct optimal trajectories connecting $q_{0}$ and $q$; namely, $q_{s}^{\prime}=\operatorname{Exp}\left(\lambda^{\prime}, s\right)$ and $q_{s}^{\prime \prime}=\operatorname{Exp}\left(\lambda^{\prime \prime}, s\right)$, $s \in[0, t]$. Moreover, $t=\mathbf{t}(\lambda)<t_{1}^{\operatorname{conj}}(\lambda)$.

(3) An optimal trajectory $q_{s}=\operatorname{Exp}(\lambda, s)$ is generated by the optimal controls

$$
u_{1}(s)=\sin \left(\gamma_{s} / 2\right), \quad u_{2}(s)=-\cos \left(\gamma_{s} / 2\right)
$$

where $\gamma_{s}$ is the solution to the equation of pendulum $\ddot{\gamma}_{s}=-\sin \gamma_{s}$ with the initial condition $\left(\gamma_{0}, \dot{\gamma}_{0}\right)=\lambda$.

Proof. For any point $q \in \widehat{M}$ there exists an optimal trajectory $q_{s}=\operatorname{Exp}(\lambda, s), s \in[0, t], \nu=(\lambda, t) \in N, \operatorname{such}$ that $q_{t}=q$ and $t \leq t_{\text {cut }}(\lambda)$. By Theorem $5.4[7]$, we have $t \leq \mathbf{t}(\lambda)$, thus $\nu \in \widehat{N}$.

(1) If $q \in \widetilde{M} \sqcup M_{\text {conj }} \sqcup M_{\text {rest }}$, then by Theorem 3.1, there exists a unique $\nu=(\lambda, t) \in \widehat{N}$ such that $q=\operatorname{Exp}(\nu)$, moreover, $\nu \in \tilde{N} \sqcup N_{\text {conj }} \sqcup N_{\text {rest }}$. Consequently, $q_{s}=\operatorname{Exp}(\lambda, s), s \in[0, t]$, is a unique optimal trajectory connecting $q_{0}$ with $q$.

The inequality $t<\mathbf{t}(\lambda)$ for $\nu=(\lambda, t) \in \widetilde{N} \sqcup N_{\text {rest }}$, and the equality $t=\mathbf{t}(\lambda)=t_{1}^{\text {conj }}(\lambda)$ for $\nu \in N_{\text {conj }}$ follow from definitions of the sets $\widetilde{N}, N_{\text {rest }}, N_{\text {conj }}$.

(2) If $q \in M_{\mathrm{Max}}$, then the statement follows similarly to item (1) from Theorem 3.1 and definition of the set $N_{\text {Max }}$.

(3) The expressions for optimal controls were obtained in Section 2 [7].

It follows from the definition of cut time that for any $\lambda \in C$ and $t \in\left(0, t_{\text {cut }}(\lambda)\right)$, the trajectory $q(s)=\operatorname{Exp}(\lambda, s)$ is optimal at the segment $s \in[0, t]$. For the case of finite $t_{\text {cut }}(\lambda)$, we obtain a similar statement for $t=t_{\text {cut }}(\lambda)$.

Theorem 3.3. If $t_{\text {cut }}(\lambda)<+\infty$, then the extremal trajectory $\operatorname{Exp}(\lambda, s)$ is optimal for $s \in\left[0, t_{\text {cut }}(\lambda)\right]$. 
Proof. Let $t_{\text {cut }}(\lambda)=\mathbf{t}(\lambda)<+\infty$, i.e., $\lambda \in C_{1} \cup C_{2} \cup C_{4}$, and let $t=t_{\text {cut }}(\lambda)$. Then $(\lambda, t) \in N_{\text {Max }}$, and the statement follows from item (2) of Theorem 3.2.

\subsection{Cut locus}

Now we are able to describe globally the first Maxwell set

$$
\operatorname{Max}=\left\{q \in M \mid \exists t>0, \exists \text { optimal trajectories } q_{s} \not \equiv q_{s}^{\prime}, s \in[0, t] \text {, such that } q_{t}=q_{t}^{\prime}=q\right\}
$$

the cut locus

$$
\text { Cut }=\left\{\operatorname{Exp}(\lambda, t) \mid \lambda \in C, t=t_{\text {cut }}(\lambda)\right\}
$$

and its intersection with caustic (the first conjugate locus)

$$
\text { Conj }=\left\{\operatorname{Exp}(\lambda, t) \mid \lambda \in C, t=t_{1}^{\mathrm{conj}}(\lambda)\right\}
$$

\section{Theorem 3.4.}

(1) $\operatorname{Max}=M_{\mathrm{Max}}$,

(2) $\mathrm{Cut}=M_{\text {cut }}$,

(3) Cut $\cap$ Conj $=M_{\text {conj }}$.

Proof. Items (1) and (2) follow from Theorem 3.2 and Corollary 3.3.

(3) Let $q \in M_{\text {conj }}$, and let $q_{s}=\operatorname{Exp}(\lambda, s), s \in[0, \mathbf{t}(\lambda)]$, be the optimal trajectory connecting $q_{0}$ with $q$. Thus there are no conjugate points at the interval $(0, \mathbf{t}(\lambda))$. By item $(1)$ of Theorem 3.2, we have $t_{1}^{\text {conj }}(\lambda)=\mathbf{t}(\lambda)$. Thus $M_{\text {conj }} \subset$ Conj, and in view of item (2) of this theorem we get $M_{\text {conj }} \subset$ Cut $\cap$ Conj. Now we prove that Cut $\cap$ Conj $\subset M_{\text {conj }}$, i.e., $M_{\text {cut }} \cap \operatorname{Conj} \subset M_{\text {conj }}$.

Fix any point $q \in M_{\text {cut }}$. Then $q=\operatorname{Exp}(\lambda, t)$ for some $(\lambda, t) \in N_{\text {cut }}=N_{\text {Max }} \sqcup N_{\text {conj. In order to complete }}$ the proof, we assume that $(\lambda, t) \in N_{\text {Max }}$ and show that $q \notin$ Conj. Since $(\lambda, t) \in N_{\text {Max }}$, then $t=\mathbf{t}(\lambda)$. We prove that $t<t_{1}^{\text {conj }}(\lambda)$.

If $\lambda \in C_{1} \cup C_{4}$, then $t_{1}^{\text {conj }}(\lambda)=+\infty$ by Theorem 2.5 [10] (see (1.14)).

Let $\lambda \in C_{2}$. If $\operatorname{sn} \tau=0$, then $(\lambda, t) \in N_{\text {conj }}$, which is impossible since $(\lambda, t) \in N_{\text {Max }}$. And if $\operatorname{sn} \tau \neq 0$, then $t<t_{1}^{\mathrm{conj}}(\lambda)$ by Proposition $2.2[10]$.

The inclusion Cut $\cap$ Conj $\subset M_{\text {conj }}$ follows.

Theorem 3.5. The cut locus has three connected components:

$$
\begin{aligned}
& \text { Cut }=\mathrm{Cut}_{\text {glob }} \sqcup \mathrm{Cut}_{\text {loc }}^{+} \sqcup \mathrm{Cut}_{\text {loc }}^{-}, \\
& \text {Cut }_{\text {glob }}=\{q \in M \mid \theta=\pi\}, \\
& \text { Cut }_{\text {loc }}^{+}=\left\{q \in M \mid \theta \in(-\pi, \pi), R_{2}=0, R_{1}>R_{1}^{1}(|\theta|)\right\}, \\
& \text { Cut }_{\text {loc }}^{-}=\left\{q \in M \mid \theta \in(-\pi, \pi), R_{2}=0, R_{1}<-R_{1}^{1}(|\theta|)\right\},
\end{aligned}
$$

where the function $R_{1}^{1}$ is defined by equation (2.20). The initial point $q_{0}$ is contained in the closure of the components $\mathrm{Cut}_{\mathrm{loc}}^{+}$, $\mathrm{Cut}_{\mathrm{loc}}^{-}$, and is separated from the component $\mathrm{Cut}_{\mathrm{glob}}$.

Proof. By Theorems 3.4, 3.1 and Lemma 2.1, we have

$$
\text { Cut }=M_{\text {cut }}=\operatorname{Exp}\left(N_{\text {cut }}\right)=\cup_{i \in C} M_{i}^{\prime} .
$$




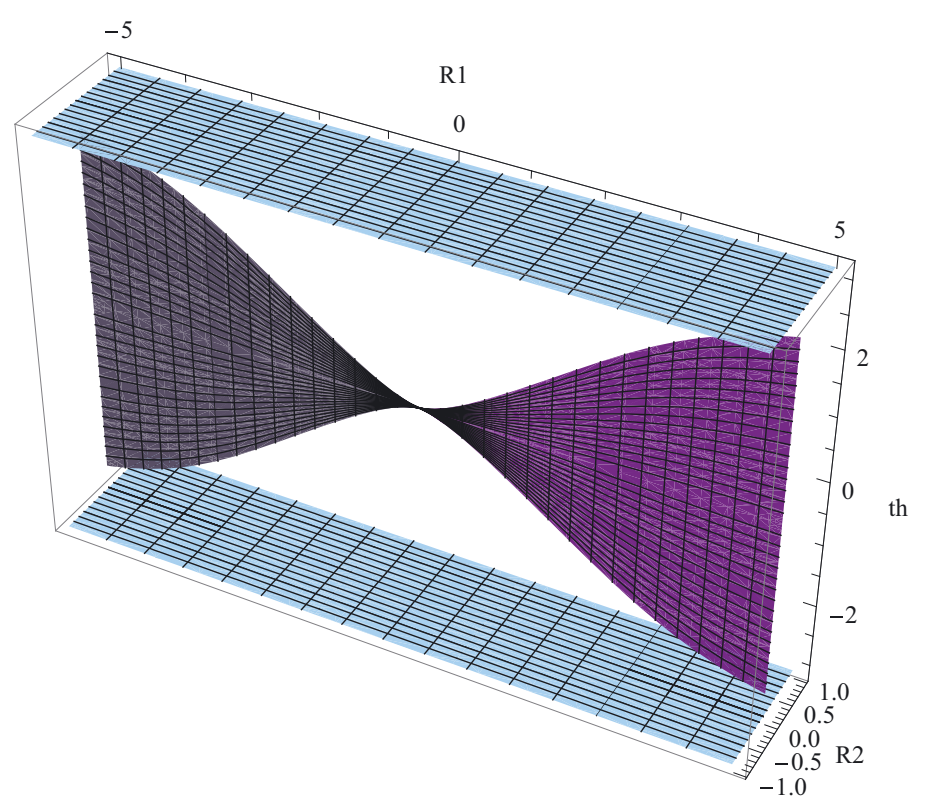

Figure 17. Cut locus in rectifying coordinates $\left(R_{1}, R_{2}, \theta\right)$.

Denote

$$
\begin{aligned}
& C_{\mathrm{glob}}=\{1, \ldots, 8,17, \ldots, 24,33,34\}, \\
& C_{\mathrm{loc}}^{+}=\{13, \ldots, 16,29, \ldots, 32\}, \\
& C_{\mathrm{loc}}^{-}=\{9, \ldots, 12,25, \ldots, 28\} .
\end{aligned}
$$

Then

$$
\cup_{i \in C_{\text {glob }}} M_{i}^{\prime}=\text { Cut }_{\text {glob }}, \quad \cup_{i \in C_{\mathrm{loc}}^{+}} M_{i}^{\prime}=\mathrm{Cut}_{\mathrm{loc}}^{+}, \quad \cup_{i \in C_{\mathrm{loc}}^{-}} M_{i}^{\prime}=\mathrm{Cut}_{\mathrm{loc}}^{-},
$$

and decomposition (3.10)-(3.13) follows.

The topological properties of $\mathrm{Cut}_{\mathrm{glob}}, \mathrm{Cut}_{\mathrm{loc}}^{ \pm}$follow from equalities (3.10)-(3.13).

The cut locus in rectifying coordinates $\left(R_{1}, R_{2}, \theta\right)$ is presented in Figure 17, notice that here the horizontal planes $\theta=0$ and $\theta=2 \pi$ should be identified. Global embedding of the cut locus to the solid torus (diffeomorphic image of the state space $M=\mathrm{SE}(2))$ is shown in Figure 18.

The curve $\Gamma=$ Cut $\cap$ Conj has the following asymptotics near the initial point $q_{0}$ :

$$
R_{1}=R_{1}^{1}(\theta)=\sqrt[3]{\pi} / 2 \theta^{2 / 3}+o\left(\theta^{2 / 3}\right), \quad \theta \rightarrow 0, \quad R_{2}=0,
$$

see item (5) of Lemma 2.5. This agrees with the result on asymptotics of cut and conjugate loci for contact sub-Riemannian structures in $\mathbb{R}^{3}$ obtained by Agrachev [1] and by El-Alaoui et al. [5].

Illustrations of cut points and the corresponding optimal trajectories are given in Figures 19-27.

\section{EXPliCit OPtimal SOLUTIONS FOR SPECIAL TERMinAL POINTS}

In this section we describe optimal solutions for particular terminal points $q_{1}=\left(x_{1}, y_{1}, \theta_{1}\right)$. Where applicable, we interpret the optimal trajectories in terms of the corresponding optimal motion of a car in the plane.

For generic terminal points, we developed a software in computer system Mathematica [13] for numerical evaluation of solutions to the problem. 


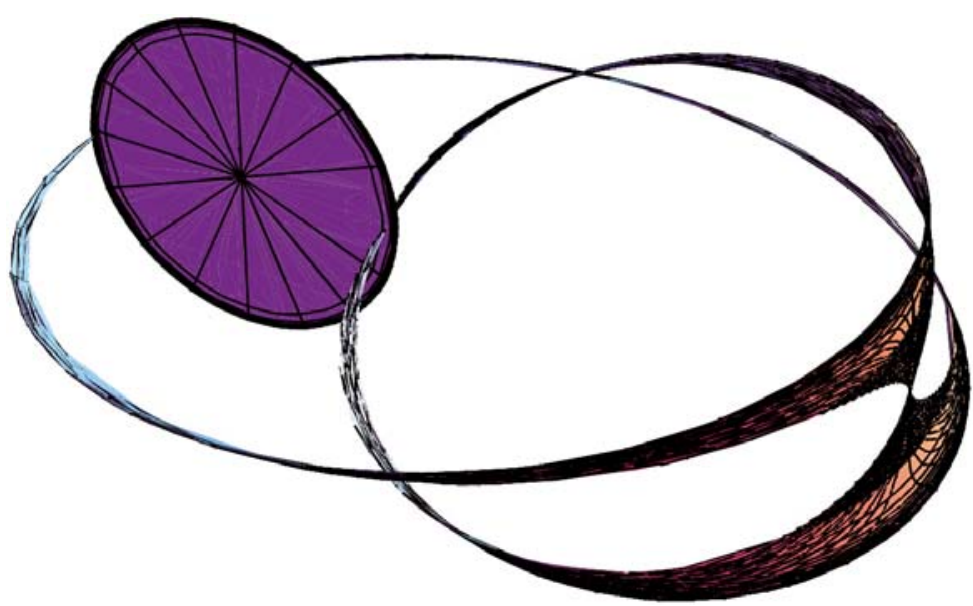

Figure 18. Cut locus: global view.

4.1. $\boldsymbol{x}_{\mathbf{1}} \neq \mathbf{0}, \boldsymbol{y}_{\mathbf{1}}=\mathbf{0}, \boldsymbol{\theta}_{\mathbf{1}}=\mathbf{0}$

In this case $\nu \in N_{5}$, and the optimal trajectory is

$$
x_{t}=t \operatorname{sgn} x_{1}, \quad y_{t}=0, \quad \theta_{t}=0, \quad t \in\left[0, t_{1}\right], \quad t_{1}=\left|x_{1}\right|,
$$

the car moves uniformly forward or backward along a segment.

4.2. $\boldsymbol{x}_{\mathbf{1}}=\mathbf{0}, \boldsymbol{y}_{\mathbf{1}}=\mathbf{0},\left|\boldsymbol{\theta}_{\mathbf{1}}\right| \in(\mathbf{0}, \boldsymbol{\pi})$

We have $\nu \in N_{4}$, and the optimal solution is

$$
x_{t}=0, \quad y_{t}=0, \quad \theta_{t}=t \operatorname{sgn} \theta_{1}, \quad t \in\left[0, t_{1}\right], \quad t_{1}=\left|\theta_{1}\right|,
$$

the car rotates uniformly around itself by the angle $\theta_{1}$.

4.3. $\boldsymbol{x}_{\mathbf{1}}=\mathbf{0}, \boldsymbol{y}_{\mathbf{1}}=\mathbf{0}, \boldsymbol{\theta}_{\mathbf{1}}=\boldsymbol{\pi}$

We have $\nu \in N_{4}$, and there are two optimal solutions:

$$
x_{t}=0, \quad y_{t}=0, \quad \theta_{t}= \pm t, \quad t \in\left[0, t_{1}\right], \quad t_{1}=\pi,
$$

the car rotates uniformly around itself clockwise or counterclockwise by the angle $\pi$, see Figure 19.

4.4. $\boldsymbol{x}_{\mathbf{1}} \neq \mathbf{0}, \boldsymbol{y}_{\mathbf{1}}=\mathbf{0}, \boldsymbol{\theta}_{\mathbf{1}}=\boldsymbol{\pi}$

There are two optimal solutions:

$$
\begin{aligned}
& x_{t}=\left(\operatorname{sgn} x_{1}\right) / k(t+E(k)-E(K+t, k)), \quad y_{t}=(s / k)\left(\sqrt{1-k^{2}}-\operatorname{dn}(K+t, k)\right), \\
& \theta_{t}=s \operatorname{sgn} x_{1}(\pi / 2-\operatorname{am}(K+t, k)), \quad s= \pm 1, \quad t \in\left[0, t_{1}\right], \quad t_{1}=2 K,
\end{aligned}
$$

and $k \in(0,1)$ is the root of the equation

$$
(2 / k)(K(k)-E(k))=\left|x_{1}\right|,
$$

see Figure 21. 


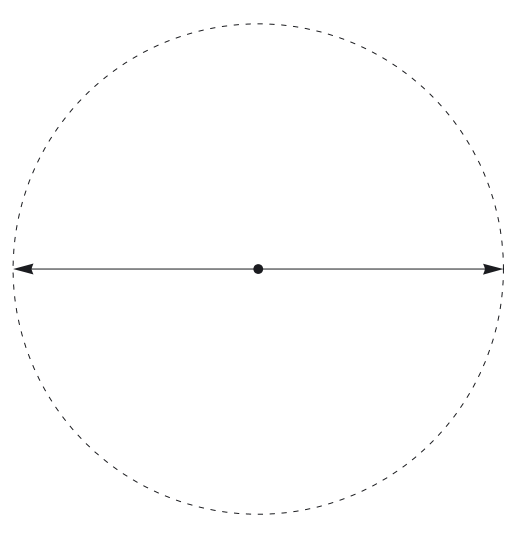

Figure 19. Cut point for $\lambda \in C_{4}$ (optimal solutions for $x_{1}=y_{1}=0, \theta_{1}=\pi$ ).

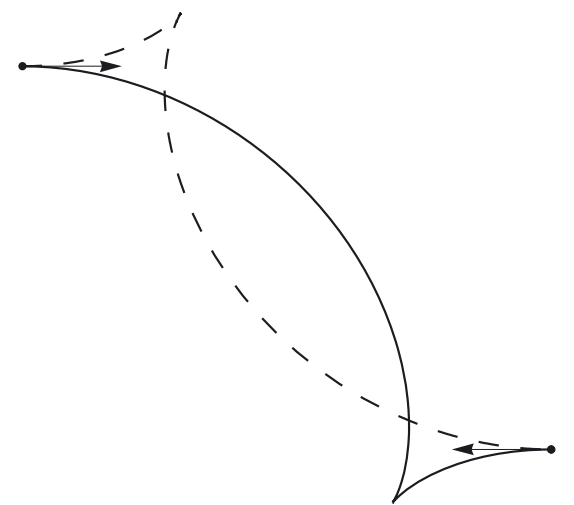

Figure 20. Cut point for $\lambda \in$ $C_{1}$, generic case (optimal solutions for $\left.\theta_{1}=\pi\right)$.

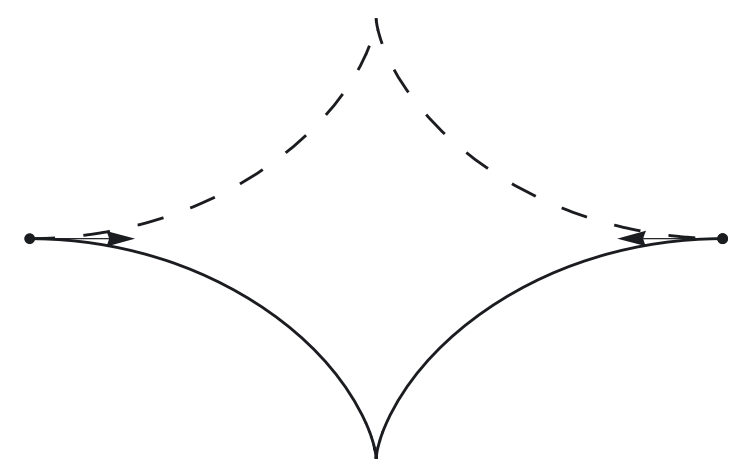

Figure 21. Cut point for $\lambda \in C_{1}$, symmetric case with cusp (optimal solutions for $x_{1} \neq 0, y_{1}=0$, $\left.\theta_{1}=\pi\right)$.

4.5. $x_{1}=0, y_{1} \neq 0, \theta_{1}=\pi$

There are two optimal solutions:

$$
\begin{aligned}
& x_{t}=s(1-\operatorname{dn}(t, k)) / k, \quad y_{t}=\left(\operatorname{sgn} y_{1} / k\right)(t-E(t, k)), \\
& \theta_{t}=s \operatorname{sgn} y_{1} \operatorname{am}(t, k), \quad s= \pm 1, \quad t \in\left[0, t_{1}\right], \quad t_{1}=2 K,
\end{aligned}
$$

and $k \in(0,1)$ is the root of the equation

$$
(2 / k)(K(k)-E(k))=\left|y_{1}\right|,
$$

see Figure 22. 


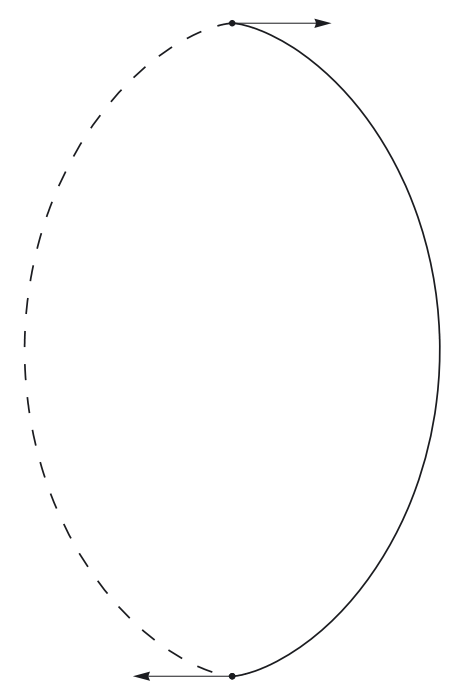

Figure 22. Cut point for $\lambda \in C_{1}$, symmetric case without cusp (optimal solutions for $x_{1}=0$, $\left.y_{1} \neq 0, \theta_{1}=\pi\right)$.

\section{6. $x_{1}=\mathbf{0}, y_{1} \neq \mathbf{0}, \theta_{1}=\mathbf{0}$}

There are two optimal solutions given by formulas for $\left(x_{t}, y_{t}, \theta_{t}\right)$ for the case $\lambda \in C_{2}$ in Section $3.3[7]$ for the following values of parameters:

$$
t \in\left[0, t_{1}\right], \quad t_{1}=2 k p_{1}^{1}(k),
$$

with the function $p_{1}^{1}(k)$ defined in Lemma 5.3 [7],

$$
s_{2}=-\operatorname{sgn} y_{1}, \quad \psi= \pm K(k)-p_{1}^{1}(k),
$$

and $k \in(0,1)$ is the root of the equation

$$
2\left(p_{1}^{1}(k)-E\left(p_{1}^{1}(k), k\right) \sqrt{1-k^{2}} / \operatorname{dn}\left(p_{1}^{1}(k), k\right)\right)=\left|y_{1}\right|,
$$

see Figure 23.

4.7. $\left(\boldsymbol{x}_{1}, \boldsymbol{y}_{1}\right) \neq \mathbf{0}, \boldsymbol{\theta}_{1}=\boldsymbol{\pi}$

Introduce the polar coordinates $x_{1}=\rho_{1} \cos \chi_{1}, y_{1}=\rho_{1} \sin \chi_{1}$. There are two optimal solutions given by formulas for $\left(x_{t}, y_{t}, \theta_{t}\right)$ for the case $\lambda \in C_{1}$ in Section 3.3 [7] for the following values of parameters:

$$
t \in\left[0, t_{1}\right], \quad t_{1}=2 K(k),
$$

and $k \in(0,1)$ is the root of the equation

$$
\begin{aligned}
& 2\left(p_{1}^{1}(k)-E\left(p_{1}^{1}(k), k\right) \sqrt{1-k^{2}} / \operatorname{dn}\left(p_{1}^{1}(k), k\right)\right)=\rho_{1}, \\
& s_{1}= \pm 1, \quad \varphi=s_{1} F\left(\pi / 2-\chi_{1}, k\right),
\end{aligned}
$$

see Figures 20-22. In the cases $y_{1}=0$ and $x_{1}=0$ we get respectively the cases considered in Sections 4.4 and 4.5. 


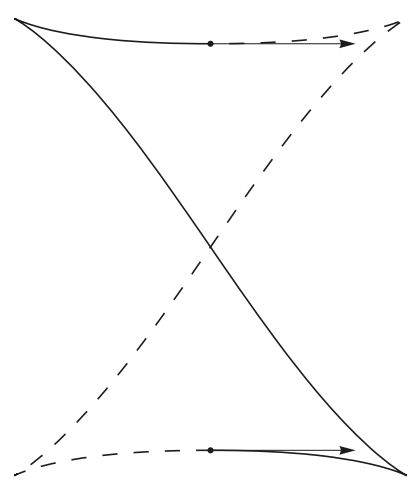

Figure 23. Optimal solutions for $x_{1}=0, y_{1} \neq 0, \theta_{1}=0$.

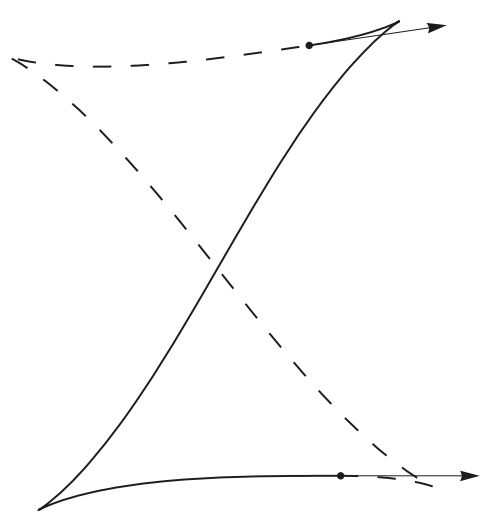

Figure 24. Cut point for $\lambda \in C_{2}$, generic case.

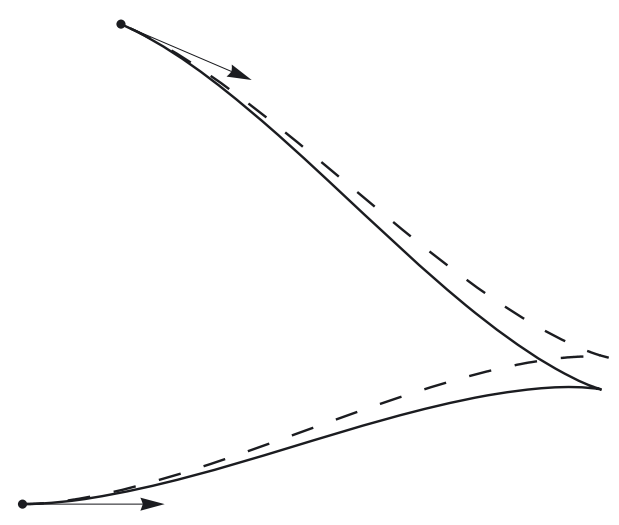

Figure 26. Cut point for $\lambda \in C_{2}$ approaching conjugate point.

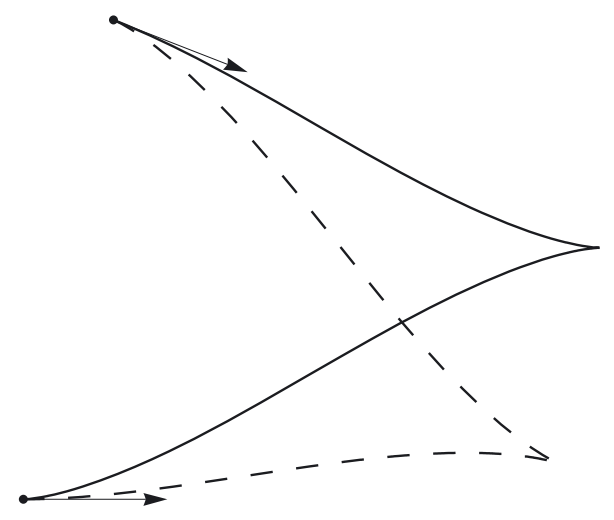

Figure 25. Cut point for $\lambda \in C_{2}$, special case with one cusp.

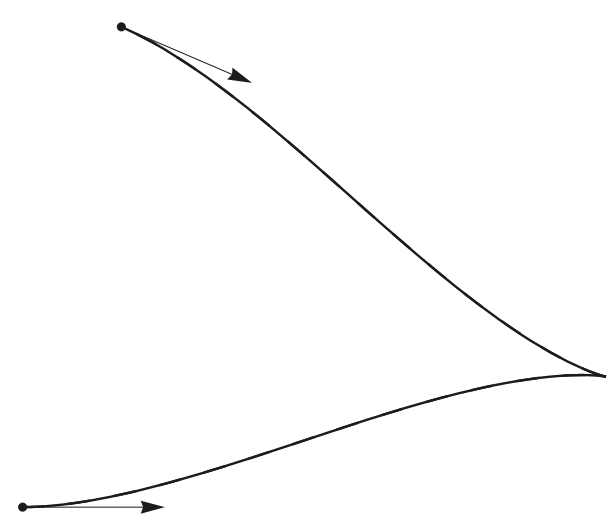

Figure 27. Cut point for $\lambda \in C_{2}$ coinciding with conjugate point. 


\section{8. $y_{1} \neq 0, \theta_{1}=0$}

There is a unique optimal solution given by formulas for $\left(x_{t}, y_{t}, \theta_{t}\right)$ for the case $\lambda \in C_{2}$ in Section 3.3 [7] for the following values of parameters:

$$
s_{2}=-\operatorname{sgn} y_{1}
$$

$k \in(0,1)$ and $p \in\left(0, p_{1}^{1}(k)\right]$ are solutions to the system of equations

$$
\begin{aligned}
& s\left(\operatorname{sgn} y_{1}\right) 2 k f_{1}(p, k) / \operatorname{dn}(p, k)=x_{1}, \quad s= \pm 1 \\
& 2(p-\mathrm{E}(p)) \sqrt{1-k^{2}} / \operatorname{dn}(p, k)=\left|y_{1}\right|
\end{aligned}
$$

and

$$
t \in\left[0, t_{1}\right], \quad t_{1}=2 k p, \quad \psi=s K(k)-p .
$$

Acknowledgements. The author is grateful to Prof. Andrei Agrachev for bringing the sub-Riemannian problem on SE(2) to authors' attention. The author wishes to thank Prof. Arrigo Cellina for hospitality and excellent conditions for work at the final version of this paper.

\section{REFERENCES}

[1] A.A. Agrachev, Exponential mappings for contact sub-Riemannian structures. J. Dyn. Control Syst. 2 (1996) 321-358.

[2] A.A. Agrachev, U. Boscain, J.P. Gauthier and F. Rossi, The intrinsic hypoelliptic Laplacian and its heat kernel on unimodular Lie groups. J. Funct. Anal. 256 (2009) 2621-2655.

[3] U. Boscain and F. Rossi, Invariant Carnot-Caratheodory metrics on $S^{3}, \mathrm{SO}(3), \mathrm{SL}(2)$, and lens spaces. SIAM J. Control Optim. 47 (2008) 1851-1878.

[4] G. Citti and A. Sarti, A cortical based model of perceptual completion in the roto-translation space. J. Math. Imaging Vis. 24 (2006) 307-326.

[5] El-H.Ch. El-Alaoui, J.-P. Gauthier and I. Kupka, Small sub-Riemannian balls on $\mathbb{R}^{3}$. J. Dyn. Control Syst. 2 (1996) 359-421.

[6] J.P. Laumond, Nonholonomic motion planning for mobile robots, Lecture Notes in Control and Information Sciences 229. Springer (1998).

[7] I. Moiseev and Yu. L. Sachkov, Maxwell strata in sub-Riemannian problem on the group of motions of a plane. ESAIM: COCV (2009) DOI: $10.1051 / \mathrm{cocv} / 2009004$.

[8] J. Petitot, The neurogeometry of pinwheels as a sub-Riemannian contact structure. J. Physiol. Paris 97 (2003) 265-309.

[9] J. Petitot, Neurogéometrie de la vision - Modèles mathématiques et physiques des architectures fonctionnelles. Éditions de l'École Polytechnique, France (2008).

[10] Yu.L. Sachkov, Conjugate and cut time in sub-Riemannian problem on the group of motions of a plane. ESAIM: COCV (2009) DOI: $10.1051 / \mathrm{cocv} / 2009031$.

[11] A.M. Vershik and V.Y. Gershkovich, Nonholonomic Dynamical Systems. Geometry of distributions and variational problems, in Itogi Nauki i Tekhniki: Sovremennye Problemy Matematiki, Fundamental'nyje Napravleniya 16, VINITI, Moscow (1987) 5-85 [in Russian]. [English translation in Encyclopedia of Math. Sci. 16, Dynamical Systems 7, Springer Verlag.]

[12] E.T. Whittaker and G.N. Watson, A Course of Modern Analysis. An introduction to the general theory of infinite processes and of analytic functions; with an account of principal transcendental functions. Cambridge University Press, Cambridge, UK (1996).

[13] S. Wolfram, Mathematica: a system for doing mathematics by computer. Addison-Wesley, Reading, USA (1991). 